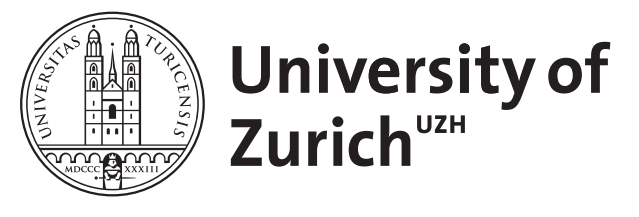

\title{
The causes of evolvability and their evolution
}

Payne, Joshua L ; Wagner, Andreas

\begin{abstract}
Evolvability is the ability of a biological system to produce phenotypic variation that is both heritable and adaptive. It has long been the subject of anecdotal observations and theoretical work. In recent years, however, the molecular causes of evolvability have been an increasing focus of experimental work. Here, we review recent experimental progress in areas as different as the evolution of drug resistance in cancer cells and the rewiring of transcriptional regulation circuits in vertebrates. This research reveals the importance of three major themes: multiple genetic and non-genetic mechanisms to generate phenotypic diversity, robustness in genetic systems, and adaptive landscape topography. We also discuss the mounting evidence that evolvability can evolve and the question of whether it evolves adaptively.
\end{abstract}

DOI: https://doi.org/10.1038/s41576-018-0069-z

Posted at the Zurich Open Repository and Archive, University of Zurich ZORA URL: https://doi.org/10.5167/uzh-166216

Journal Article

Accepted Version

Originally published at:

Payne, Joshua L; Wagner, Andreas (2019). The causes of evolvability and their evolution. Nature Reviews. Genetics, 20(1):24-38.

DOI: https://doi.org/10.1038/s41576-018-0069-z 


\section{The causes of evolvability and their evolution}

2 Joshua L. Payne ${ }^{1}$ and Andreas Wagner ${ }^{2}$

3 1. Institute of Integrative Biology, ETH Zurich, Zurich, 8092, Switzerland

4 2. Department of Evolutionary Biology and Environmental Studies, University of Zurich, 8092, Switzerland

5 Correspondence to A.W.

6 e-mail: andreas.wagner@ieu.uzh.ch

7

8 Abstract | Evolvability is the ability of a biological system to produce phenotypic variation that is both

9 heritable and adaptive. It has long been the subject of anecdotal observations and theoretical work. In recent

10 years, however, the molecular causes of evolvability have been an increasing focus of experimental work.

11 Here we review recent experimental progress in areas as different as the evolution of drug resistance in

12 cancer cells and the rewiring of transcriptional regulation circuits in vertebrates. This research reveals three

13 major themes: the importance of multiple, genetic and non-genetic mechanisms to generate phenotypic

14 diversity, of robustness in genetic systems, and of adaptive landscape topography. We also discuss the

15 mounting evidence that evolvability can evolve, and the question of whether it evolves adaptively.

17 [H1] Introduction

18 Evolvability research is now entering its fourth decade. Although the term was first used as early as 1932,

19 evolvability as a scientific subdiscipline of evolutionary biology is often associated with a 1989 article by

20 Richard Dawkins ${ }^{1}$ describing what are now called digital organisms ${ }^{2}$. Today, research on evolvability is

21 integral to multiple fields, including population genetics, quantitative genetics, molecular biology, and

22 developmental biology. Not surprisingly then, this diversity of research has led to various definitions of

23 evolvability ${ }^{3}$. We here focus on one of them, because we consider it the most fundamental: Evolvability is the

24 ability of a biological system to produce phenotypic variation that is both heritable and adaptive. The

25 definition is fundamental because, first, heritable phenotypic variation is the essential raw material of

26 evolution. Second, unless a biological system has the potential to produce variation that is adaptive 
27 (beneficial) in some environments, adaptation by natural selection is impossible. Third, the definition is broad 28 enough to apply to fields as different as population genetics and molecular biology, which study evolvability 29 in different ways ${ }^{3}$.

31 Most early evolvability research was theoretical or guided by few experimental studies ${ }^{1,3-11}$. This has changed.

32 Research on evolvability is becoming increasingly experimental and driven by advances in high-throughput 33 technologies (Box 1). The observations from such experiments are providing a mechanistic understanding of 34 how living systems generate heritable adaptive variation ${ }^{12}$. We focus this Review on such experimental 35 studies, which come from a diversity of fields, ranging from developmental to cancer biology. Many make no 36 explicit mention of evolvability, yet they all shed light on the causes of evolvability, and some also on its 37 evolution. They are relevant for phenomena as different as the evolution of antibiotic resistance in bacteria, 38 and the evolutionary rescue of populations threatened by climate and other environmental change. Their 39 insights fall into three major categories, which provide a scaffold for this Review.

41 The first major category encompasses molecular mechanisms that create phenotypic heterogeneity, and do so 42 not just through DNA mutations, but even in the absence of such mutations. These mechanisms have become 43 central to evolvability research, because they allow isogenic populations [G] to create phenotypic variation, 44 some of which may facilitate survival in new or rapidly changing environments, and may thus provide time 45 for an advantageous phenotype to be reinforced or stabilized via DNA mutation, gene duplication, 46 recombination, or epigenetic modification. The second category of evidence revolves around robustness, 47 which is central to evolvability, because it allows an evolving population to explore new genotypes without 48 detrimentally affecting essential phenotypes. The resulting genotypic diversity may serve as a springboard for 49 subsequent mutations to generate novel phenotypes, or it may bring forth new phenotypic variation when the 50 environment changes. The third category of evidence regards the topographical features of an adaptive 51 landscape, such as its smoothness, and a population's location within such a landscape. These factors 52 determine the amount of adaptive phenotypic variation that mutation can bring forth. Adaptive landscapes 
53 provide a useful geometric framework to encapsulate genotype-phenotype (or fitness) relationships that affect 54 evolvability.

55

56 Unfortunately, space constraints prevent us from reviewing other important aspects of evolvability research, 57 including the roles of phenotypic plasticity [G], organismal development, modularity [G], and pleiotropy 58 [G], as well as theoretical advances. Additionally, we frame our Review primarily around mechanisms of 59 pre-mutation evolvability [G] and mechanisms that do not require genetic change, although we briefly 60 discuss some mechanisms of post-mutation evolvability [G], where recombination plays an especially 61 important role ${ }^{13}$.

62

\section{3 [H1] Phenotypic heterogeneity}

64 Heritable phenotypic variation is the raw material of natural selection, and the best-known mechanisms to 65 create such variation are DNA mutation and recombination. However, because the role these mechanisms 66 play in generating phenotypic variation is well established and has been extensively reviewed ${ }^{13,14}$, we here 67 focus on another class of mechanisms whose astonishing diversity is only beginning to come to light through 68 recent experimental work ${ }^{15}$. These mechanisms create phenotypic heterogeneity without creating genetic 69 variation.

71 Non-genetic mechanisms to create phenotypic heterogeneity can be found in many processes affecting the 72 expression of genetic information. We review four such mechanisms: stochastic gene expression, errors in 73 protein synthesis, epigenetic modifications, and protein promiscuity. Each mechanism can create phenotypic

74 variation in a population of genetically identical individuals ${ }^{16}$. Such variation can for example provide a

75 competitive advantage to subpopulations with adaptive phenotypes in fluctuating environments ${ }^{17,18}$. These

76 phenotypes may themselves be heritable, eventually made permanent by mutation or epigenetic modification, 77 or they may simply 'buy time' for a population to adapt in other ways to an environmental challenge (Fig. 78 1a). 
80 [H2] Stochastic gene expression. Stochastic gene expression, or gene expression noise [G] has multiple

81 causes, including the efficiency of transcription and translation ${ }^{19,20}$, as well as the regulation of gene

82 expression by low-abundance molecules whose numbers fluctuate randomly in a cell ${ }^{21}$ (Fig. 1b). It can create

83 non-genetic, adaptive diversity in phenotypes as diverse as viral latency [G] , bacterial competence [G] and

84 antibiotic resistance, as well as drug resistance in cancer ${ }^{22-24}$.

86 One example where stochastic gene expression causes adaptive phenotypic variation is persistence, where

87 some cells in an isogenic population exhibit a physiologically dormant phenotype called a persister

88 phenotype $\mathrm{e}^{25}$. This phenotype is adaptive, because a dormant subpopulation has the potential to survive drugs

89 that require active growth for killing, affording the persistent subpopulation time to acquire resistance-

90 conferring DNA mutations. This was recently demonstrated in a laboratory evolution experiment of

91 Escherichia coli populations subjected to intermittent exposures of ampicillin ${ }^{26}$, in which persistence served

92 as a stopgap until some individuals acquired resistance-causing mutations.

94 Persistence arises in only a small fraction of a population, so one might think that the resulting population

95 bottleneck [G] would hinder evolvability by reducing the supply of beneficial mutations. However, a recent

96 study of non-small-cell lung cancer indicates that this need not be the case $\mathrm{e}^{27}$. These cells stochastically

97 express a persistent phenotype, mediated by an altered chromatin state ${ }^{28}$. A population derived from one of

98 these cells was exposed to the drug erlotinib, which resulted in the formation of multiple persistent

99 subpopulations. Seventeen of these subpopulations were later expanded in isolation from each other until

100 drug resistance emerged through DNA mutations. Genetic analysis of the resistant clones uncovered several

101 distinct resistance mechanisms, indicating that several evolutionary paths to resistance remained despite the

102 population bottleneck. In sum, persistence can facilitate evolvability, because it allows some individuals

103 (individual cells in this example) to survive long enough to experience adaptive genetic change. 
105 Rare-cell-variability is similar to persistence, in that a subpopulation of cells stochastically expresses a 106 phenotype that facilitates the evasion of drug treatment ${ }^{28,29}$. It is different from persistence, in that the 107 subpopulation is not dormant, but rather exhibits a transient transcriptional state that may include the 108 expression of resistance-conferring genes. For example, in a study of resistance evolution to the drug 109 vemurafenib in human melanoma, rare cells transiently expressed one or several such genes prior to drug

110 exposure, making them 'pre-resistant'. ${ }^{24}$ After four weeks of drug exposure, stably resistant colonies emerged

111 that expressed these genes at uniformly high levels, and in a semi-coordinated fashion. For instance, of 1,456

112 genes known to contribute to resistance, pre-resistant cells expressed 72. After four weeks of drug exposure, 113 this number rose to 966 . These changes were not caused by DNA mutations. Rather, drug exposure initiated 114 epigenetic cellular changes that stabilized the transiently resistant state. The transient expression of

115 resistance-conferring genes in rare cells is not limited to melanoma, but is also found in unrelated cancer cell

116 types, suggesting that the epigenetic conversion of a rare, transient transcriptional state to a stably resistant 117 state may be a common mechanism of evolvability in cancer ${ }^{30}$. Such stabilization of a new phenotype, even if 118 temporary, may facilitate more permanent stabilization through genetic mutations. Examples like these are 119 closely related to the phenomenon of genetic assimilation [G] , which has been studied since the $1950 \mathrm{~s}^{31,32}$.

121 Stochastic gene expression may also facilitate evolvability by changing how strongly mutations affect fitness, 122 and in particular by enhancing the positive effects of beneficial mutations ${ }^{33}$. This was recently demonstrated

123 using synthetic gene circuits in Saccharomyces cerevisiae ${ }^{34}$, which were engineered to exhibit varying 124 degrees of expression heterogeneity in an antifungal resistance gene. Populations harbouring a version of a 125 circuit with high expression heterogeneity were compared to those harbouring a circuit with low expression 126 heterogeneity. During an evolution experiment where populations were exposed to increasing concentrations 127 of the antifungal drug fluconazole, high-heterogeneity populations went extinct less often and evolved higher

128 fluconazole resistance than low-heterogeneity populations. At least partly responsible were the increased 129 beneficial effects of flucanozole resistance mutations in high-heterogeneity populations, because the same 
130 resistance mutations conferred greater resistance when expressed with high expression heterogeneity than

131 with low heterogeneity. Altering the phenotypic effects of mutations is therefore another route by which

132 stochastic gene expression can facilitate evolvability ${ }^{33}$.

133

134 [H2] Errors in protein synthesis. In addition to stochastic gene expression, protein synthesis errors can also

135 create non-genetic phenotypic heterogeneity. Such errors come in many forms and occur at multiple stages of

136 protein synthesis, including nucleotide misincorporation during transcription, tRNA misacylation during

137 translation, and kinetic trapping [G] during protein folding ${ }^{35}$. Translation is particularly error-prone, with

138 rates of mistranslation exceeding those of DNA point mutations by several orders of magnitude. Such errors

139 are also called phenotypic mutations ${ }^{36}$, and they include missense, read-through, and frameshift mutations.

140 Phenotypic mutations can facilitate evolvability, because they create variation in a protein pool expressed

141 from the same gene, and some of this variation may be adaptive (Fig. 1c). For example, elevated

142 mistranslation rates in Mycobacterium tuberculosis generate variation in the beta subunit of RNA

143 polymerase, which increases resistance to the antibiotic rifampicin ${ }^{37}$. Similarly, mistranslation of CUG

144 codons in the fungal pathogen Candida albicans generates variation in cell surface proteins that facilitate

145 evasion of the host's immune system ${ }^{38}$.

146

147 A special kind of mistranslation error is stop-codon readthrough [G], which is a common mechanism for

148 generating protein variation in species as different as yeast, fly and human ${ }^{39,40}$. In fungi, for example, it can

149 lead to the expression of cryptic peroxisomal signalling motifs that create variation in the cellular localization

150 of proteins ${ }^{40}$. In crustacea and hexapods, DNA sequences downstream of an affected stop codon are often

151 evolutionarily conserved, suggesting that stop-codon readthrough occurs frequently enough to affect the

152 evolution of cryptic sequences ${ }^{41,42}$.

153 
154 Protein synthesis errors not only enhance evolvability by increasing protein diversity, they can also help pave

155 the way for subsequent adaptive genetic change ${ }^{43,44}$. An example comes from the S. cerevisiae protein IDP3,

156 an NADP-dependent isocitrate dehydrogenase that localizes to the peroxisome ${ }^{45}$. The protein originated in an

157 ancient yeast whole-genome duplication, and diverged from its cytosolic ancestor IDP2 by acquiring a C-

158 terminal peroxisomal targeting signal, while IDP2 remained cytosolic. Yeast species that diverged before the

159 whole-genome duplication possess only a cytosolic IDP2 gene, but in four of these species the gene contains

160 a cryptic peroxisomal targeting signal in the $3^{\prime}$ untranslated region. This signal can be revealed via a +1

161 translational frameshift that bypasses the stop codon, which exposes the mistranslated protein to selection for

162 peroxisomal targeting and function, and can, for example, lead to an increase in the strength of the

163 peroxisomal signalling motif ${ }^{45}$. The frameshift is induced by a sequence context that is prone to ribosomal

164 slippage, and that is also prone to single nucleotide deletions that mimic the effect of the frameshift on

165 protein sequence. This correlation between phenotypic and genotypic mutations thus facilitated the evolution

166 IDP3: Before the whole-genome duplication, IDP2 could already be expressed in two locations: in the

167 cytosol through faithful translation, and in the peroxisome through mistranslation. After the whole-genome

168 duplication, the peroxisomal localization and function was made permanent via a single base deletion in one

169 of the gene copies.

170

171 [H2] Epigenetic modifications. Phenotypic heterogeneity can also be caused by epigenetic changes, such as

172 methylation of DNA and histones, alteration of chromatin structure, and the changes in protein conformation

173 known as prions [G]. For example, the prion $\left[\mathrm{PSI}^{+}\right]$in $S$. cerevisiae is an aggregated conformation of the

174 translational suppressor protein Sup35, which can be inherited by forming inactive complexes that convert

175 other Sup35 proteins to the same inactive state ${ }^{18}$. Such aggregation reduces translational fidelity, which

176 causes translational errors that include stop-codon readthrough events and frameshifts in other proteins ${ }^{46}$ (Fig.

177 1d). Some of these errors reveal cryptic genetic variation [G] , producing phenotypes that are heritable and

178 that can be adaptive ${ }^{18,47}$. For example, $\left[\mathrm{PSI}^{\dagger}\right]$ can improve growth on a variety of carbon and nitrogen sources,

179 and in various temperatures and stress conditions ${ }^{18,48}$. The phenotypes induced by $\left[\mathrm{PSI}^{+}\right]$and other prions can 
180 persist for generations, which provides opportunity for the phenotypes to be reinforced by mutation or

181 recombination, or to interact with existing genetic variation or new mutations to form novel, potentially

182 adaptive phenotypes ${ }^{47,49}$. Recent research in this area has greatly expanded the repertoire of known prions ${ }^{49-51}$,

183 elucidated the mechanisms by which they confer a selective advantage ${ }^{52-54}$, and uncovered alternative forms

184 of protein-based inheritance ${ }^{55-57}$. For instance, the first bacterial prion has recently been identified ${ }^{50}$. It is the

185 transcription terminator Rho of Clostridium botulinum, which can take on one of two conformations, a

186 soluble form that does not impact transcription, and an aggregate prion form that can self-propagate and that

187 alters transcription, causing genome-wide transcriptomic changes. Its discovery raises the exciting possibility

188 that this cause of evolvability is ancient and predates the origin of eukaryotes.

190 The methylation of DNA and histones are heritable epigenetic modifications, which create phenotypic

191 variation that can be adaptive ${ }^{58,59}$. A recent example comes from the study of intra-tumour heterogeneity in

192 cancer $^{60}$. Proliferative potential varies among cancer cells within the same tumour, and those cells that

193 preserve proliferative potential can drive long-term tumour growth. Some of this variation is caused by an

194 epigenetic modification to an enhancer [G] that modulates the expression of the linker histone H1.0, which

195 is involved in the compaction of chromatin. Specifically, DNA methylation of the enhancer represses the

196 expression of the linker histone. This destabilizes nucleosome-DNA interactions, which de-represses the

197 expression of oncogenes that support proliferative potential. Thus, variation in the epigenetic modification of

198 a regulatory element creates variation in chromatin structure, some of which facilitates cancer cell self-

199 renewal. This epigenetic cause of intra-tumour heterogeneity is found in dozens of cancers ${ }^{60}$, and it is just one

200 of several epigenetic causes of phenotypic heterogeneity in this disease ${ }^{59}$.

201

202 [H2] Protein promiscuity. A fourth cause of evolvability-enhancing phenotypic heterogeneity is protein

203 promiscuity ${ }^{61,62}$. Promiscuous proteins have one primary adaptive function and other secondary latent

204 functions. Prominent examples include enzymes with 'moonlighting' catalytic activities ${ }^{63,64}$, such as bacterial 
205 carbonic anhydrase II, which mainly catalyzes the reversible hydration of carbon dioxide, but also exhibits 206 promiscuous activity toward esters ${ }^{61}$. Promiscuity can facilitate evolvability, because it provides a reservoir 207 of potentially adaptive protein activities that can be enhanced by gene duplication, when such duplications 208 are followed by mutations that refine different activities in different duplicates. For example, in S. cerevisiae, 209 two transcription factors that are products of a past gene duplication regulate the genes involved in maltose

210 metabolism and the genes involved in palatinose metabolism ${ }^{65}$. These duplicates arose from a single

211 promiscuous transcription factor that regulated the expression of both the maltose- and palatinose-specific

212 genes. After gene duplication, two single-nucleotide mutations in the DNA binding domain of one of the

213 duplicates altered its binding specificity, such that it could no longer bind the promoters of the maltose-

214 specific genes. Mutations in the coding region of the other duplicate weakened its activity toward maltose,

215 such that it could only activate the maltose-specific genes, because their promoters contained multiple

216 binding sites for the protein, which compensated for its reduced activity. Gene duplication thus facilitated the

217 partitioning of the promiscuous activity of a single transcription factor among its duplicates.

219 Sometimes duplication may not even be needed to reinforce a promiscuous function ${ }^{66,67}$. This is especially 220 true for regulatory elements. For example, the Drosophila santomea gene Neprilysin-1 evolved a novel 221 expression pattern in the fly's optic lobe via a small number of mutations to an existing enhancer ${ }^{68}$.

222 Reconstruction of the enhancer's ancestral state revealed its promiscuous activity in the optic lobe, indicating 223 that these mutations did not generate new enhancer activity de novo, but rather refined one of the enhancer's 224 existing, latent activities.

226 In sum, these examples show how various forms of phenotypic heterogeneity — caused by stochastic gene 227 expression, errors in protein synthesis, epigenetic modifications, and protein promiscuity — facilitate the 228 exploration of novel phenotypes. Some of these phenotypes may be adaptive, and may be made permanent by 229 selection for genetic or epigenetic changes that reinforce the phenotype. We emphasize that many other 
mechanisms to regulate molecular processes exist, and given the adaptive benefits of phenotypic

231 heterogeneity, it is likely that they will also be implicated in producing such heterogeneity.

\section{3 [H1] Robustness}

234 Robustness to DNA mutations can be viewed as a dual, converse, or opposite property to non-genetic

235 phenotypic heterogeneity. Whereas non-genetic phenotypic heterogeneity implies that phenotypic variation

236 exists in the absence of genetic variation, robustness implies that phenotypic variation does not exist in the

237 presence of genetic variation, because a phenotype is robust to genetic change.

239 Many phenotypes are to some extent robust to mutations ${ }^{69,70}$. Examples include the structure and biological

240 activity of macromolecules ${ }^{71}$, the gene expression patterns of regulatory networks ${ }^{72}$, and the ability of a

241 metabolism to synthesize biomass ${ }^{73}$. Such robustness can also be enhanced in various ways. For example,

242 DNA mutations that enhance protein stability can also enhance robustness, because enhanced protein stability

243 increases the range of mutations a protein can experience while still folding into its native structure ${ }^{71}$. Gene

244 duplication can also enhance robustness, because it causes gene functions to become redundant, and can thus

245 increase the incidence of mutations that can be tolerated by either duplicate ${ }^{74}$ (but see refs ${ }^{75,76}$ ). Chaperones

246 [G] such as the eukaryotic protein Hsp90 enhance robustness in organisms as diverse as fruit flies, cave fish,

247 plants and bacteria ${ }^{77-82}$, although such buffering may not occur in all organisms and may not affect all genetic 248 variation $^{78,83}$.

250 In each of these cases, DNA mutations can cause genetic diversity without changing a phenotype. Such

251 cryptic genetic variation can facilitate evolvability in at least three ways. First, cryptic genetic variation may

252 be revealed as phenotypic variation, for example via the partial loss of function of a chaperone or via the

253 appearance of a prion, or when the environment changes ${ }^{18,42,47,78,81,84,85}$. Because these phenotypes are

254 occasionally exposed to selection, cryptic genetic variation may be enriched for adaptations ${ }^{42}$. Second, cryptic 
255 genetic variation provides many distinct genetic backgrounds in which the effects of new mutations can

256 manifest themselves ${ }^{86,87}$. This can be advantageous because the same mutation can have different phenotypic

257 effects - neutral, beneficial, or detrimental — in different genetic backgrounds, a phenomenon caused by

258 frequent epistatic interactions [G] (non-additive interactions) among mutations. Finally, cryptic genetic

259 variation may give rise to new phenotypic variation via recombination.

260

261 The study of robustness has a long history in evolvability research ${ }^{69,88}$, but recent experimental work has

262 greatly expanded our mechanistic understanding of how robustness facilitates the generation of adaptive

263 phenotypic variation. These advances largely result from technological progress in areas such as deep

264 mutational scanning and ancestral protein reconstruction (Box 1). We highlight recent examples from

265 individual macromolecules, from interactions between macromolecules and their ligands, and from entire

266 gene regulatory networks.

267

268 The $\mathrm{C} 2 \mathrm{H} 2$ zinc finger is the most prominent protein domain [G] in many metazoans, but not in other

269 eukaryotes. It occurs in $\mathrm{C} 2 \mathrm{H} 2$ zinc finger transcription factors, where multiple copies of this domain are

270 typically arranged in tandem, such that each domain contacts three or more DNA bases, the identity of which

271 is determined by four base-contacting amino acids in the domain's alpha helix. The diversity of DNA

272 sequences recognized by metazoan $\mathrm{C} 2 \mathrm{H} 2$ zinc fingers far exceeds that of other eukaryotic $\mathrm{C} 2 \mathrm{H} 2$ zinc fingers,

273 and recent research implicates robustness in their expansion and diversification ${ }^{89}$. Specifically, in metazoans,

274 non-base-contacting amino acids of the $\mathrm{C} 2 \mathrm{H} 2$ zinc finger domain form hydrogen bonds with the DNA

275 phosphate backbone to enhance binding energy. By contrast, the binding energy of other eukaryotic $\mathrm{C} 2 \mathrm{H} 2$

276 zinc fingers depends primarily on base-contacting amino acids. This suggests that the non-base-contacting

277 amino acids of metazoan $\mathrm{C} 2 \mathrm{H} 2$ zinc fingers confer robustness of DNA binding to mutations in base-

278 contacting amino acids, which facilitates the diversification of DNA binding preferences. 
280 The evolution of steroid receptor binding preferences provides another example of how robustness facilitates 281 evolvability. Steroid receptors are transcription factors that can be classified according to their binding 282 preference for oestrogen response elements or steroid response elements. These two response elements are 283 6nt-long DNA sequences that differ by just two nucleotides. The ancestral steroid receptor from which all 284 steroid receptors descended more than 450 million years ago binds oestrogen response elements ${ }^{90}$. After this 285 protein duplicated, one daughter protein retained specificity to oestrogen elements, whereas the other evolved 286 a preference for steroid response elements. This shift in specificity required eleven substitutions outside of 287 the DNA binding domain and three substitutions within it. The eleven mutations outside of the DNA binding 288 domain did not affect DNA binding specificity — specificity was robust to genetic changes — but they had 289 another important consequence: they dramatically altered the number of mutational variants capable of 290 binding steroid response elements. Specifically, out of 160,000 possible mutational variants of the ancestral 291 protein without the 11 mutations, only 41 specifically bound steroid response elements. By contrast, of the 292 same 160,000 mutational variants of the ancestral protein with the 11 mutations, 829 specifically bound 293 steroid response elements, and these variants were accessible via fewer mutations ${ }^{91}$. The mutational 294 neighbourhoods of the two proteins were therefore dramatically different, and it was the robustness to 295 mutation that facilitated access to the mutational neighbourhood that conferred higher evolvability (Fig. 2).

297 Not only are regulatory proteins robust to mutation, so too are the regulatory elements they target ${ }^{87,92}$. For 298 example, eukaryotic transcription factors typically bind dozens to hundreds of distinct nucleic acid 299 sequences ${ }^{93}$, which tend to be mutationally interconnected, such that a mutation to a sequence that binds a 300 transcription factor will often generate another sequence that also binds the transcription factor ${ }^{87}$. This 301 robustness facilitates the accumulation of genetic diversity in binding sites ${ }^{94}$, which provides distinct genetic 302 backgrounds in which to test new mutations. Some of these mutations generate binding sites for other 303 transcription factors ${ }^{87}$, which may lead to adaptive gene expression changes. 
305 Gene expression patterns themselves are highly robust, not only to mutations in binding sites, but also to 306 wholesale changes in the number, identity, and orientation of binding sites within regulatory regions ${ }^{95}$, and 307 thus to changes in the structure of gene regulatory networks ${ }^{96}$. Modelling work has long anticipated that such 308 robustness can facilitate evolvability ${ }^{97,98}$, but empirical support for this possibility was only recently

309 provided $^{99}$. Specifically, the highly conserved fungal transcription factor Ndt80 underwent a pronounced 310 switch in function from an ancestral role regulating meiosis and sporulation to a derived role regulating

311 biofilm formation. Experiments with six different extant yeast species suggest that this shift was not caused

312 by a change in the binding specificity of $N d t 80$, but rather by gains and losses of binding sites for $N d t 80$.

313 These changes preserved the ancestral role of $N d t 80$ but allowed the regulatory network controlling meiosis

314 and sporulation to sample many architectural configurations. This sampling facilitated the discovery of a

315 network configuration that supported the derived role of biofilm production in Candida albicans.

317 In sum, these examples illustrate that robustness creates opportunities for the exploration of novel genotypes,

318 some of which constitute or lead to new adaptations. Other pertinent examples include recent studies of

319 robustness in viral proteins ${ }^{100,101}$, bacterial enzymes ${ }^{102}$, tumour suppressor genes ${ }^{103}$, protein-protein

320 interactions ${ }^{104,105}$ and gene regulatory networks ${ }^{106}$.

\section{2 [H1] Adaptive landscape topography}

323 An adaptive landscape is an analogy to a physical landscape, in which each location or coordinate in a 324 physical space corresponds to a genotype in an abstract genotype space [G] ${ }^{107}$, and where the elevation at 325 this location corresponds to the fitness of this genotype ${ }^{108}$. One can view adaptive evolution as a process 326 where populations of ever-changing genotypes explore such a landscape through random DNA mutations and recombination, and where natural selection helps such populations discover peaks or plateaus of high fitness. Adaptive landscapes are central to evolvability research, because the topography of an adaptive landscape, and a population's location within a landscape, determine the amount of beneficial phenotypic variation that 
330 mutations can create. A smooth, single-peaked landscape facilitates evolvability, because mutation can bring 331 forth beneficial phenotypic variation from anywhere in the landscape, except atop a global peak (Fig. 3a). In 332 contrast, a rugged landscape can hinder evolvability, because the local peaks it contains may attract an 333 evolving population and preclude the generation of further beneficial phenotypic variation (Fig. 3b). 334 Moreover, the shape of an adaptive peak - concave [G] versus convex [G] - affects the amount of 335 beneficial phenotypic variation that mutation can bring forth as an evolving population ascends the peak. 336 Until recently, most work on adaptive landscapes was theoretical, but experiments are now being increasingly 337 used to characterize the topography of adaptive landscapes ${ }^{109}$. Some of these studies use organismal fitness to 338 define the surface of a landscape $e^{110,111}$, whereas others use molecular phenotypes, such as the enzymatic 339 activity ${ }^{112,113}$ or binding affinity ${ }^{114,115}$ of a protein, and are therefore also referred to as genotype-phenotype 340 landscapes ${ }^{116}$. The pace of this work is still accelerating, and we focus on the most recent such work.

342 Perhaps the most important factor affecting landscape ruggedness and the shape of adaptive peaks is epistasis 343 - non-additive interactions among two or more mutations ${ }^{117,118}$. Epistasis can take different forms (Fig.

$3443 \mathrm{c}, \mathrm{d})$, and can occur with mutations that are individually deleterious or beneficial. For example, negative 345 epistasis amongst beneficial mutations occurs when the combined effect of the mutations is smaller than the 346 sum of the individual mutational effects ${ }^{119,120}$ (Fig. 3c). It is also referred to as antagonistic or diminishing 347 returns epistasis. Positive epistasis amongst beneficial mutations occurs when the combined effect of the 348 mutations is larger than the sum of the individual mutational effects (Fig. 3c). It is also referred to as 349 synergistic epistasis. The terminology used to describe epistasis can be confusing (e.g., synergistic epistasis is 350 also used to describe negative epistasis amongst deleterious mutations ${ }^{121}$ ), but mathematically the definition 351 of positive and negative epistasis is straightforward. Epistasis amongst two mutations $A$ and $B$ can be 352 quantified as $\varepsilon=f_{\mathrm{ab}}+f_{\mathrm{AB}}-f_{\mathrm{Ab}}-f_{a \mathrm{~B}}$, where $f$ is the phenotype or fitness of the 'wild type', double mutant, and 353 single mutant genotypes, respectively. Negative epistasis occurs when $\varepsilon<0$, whereas positive epistasis occurs 354 when $\varepsilon>0$. 
356 Another important form of epistasis is sign epistasis ${ }^{122}$. It occurs when the sign — beneficial $(+)$ or

357 detrimental (-) - of a double mutation differs from that of one or both of the constituent single mutations.

358 For example, whereas both single mutations may be individually detrimental, they may be jointly beneficial.

359 Sign epistasis creates local valleys or peaks and thus ruggedness in an adaptive landscape (Fig. $3 \mathrm{~d})^{118}$. In

360 doing so, it can affect the amount of adaptive variation accessible to a population, a population's evolutionary

361 trajectory, and its ability to reach a global peak. For example, global peaks may be inaccessible if all

362 evolutionary trajectories to them require traversing one or more adaptive valleys, which is disfavoured by

363 natural selection and possible only under restricted conditions ${ }^{123,124}$. With some exceptions ${ }^{125-127}$, sign

364 epistasis thus reduces evolvability.

366 A fundamental challenge in mapping an adaptive landscape is that the number of genotypes in a typical 367 genotype space is so vast that their phenotype or fitness cannot usually be exhaustively measured. One 368 approach to overcome this challenge uses experimental evolution of whole organisms ${ }^{128}$, where the change in

369 a population's mean fitness and genotypic composition is monitored while the population evolves for

370 hundreds or thousands of generations in the laboratory. Such experiments show that even though specific 371 genetic changes that cause fitness increases are usually not predictable, the evolutionary trajectory of mean 372 fitness increases can be highly predictable ${ }^{129-132}$, suggesting that suitable statistical methods may be able to 373 infer general statistical properties of adaptive landscape topography ${ }^{133,134}$. Additionally, experimental 374 evolution demonstrates that a population's mean fitness increase - a proxy for evolvability — depends 375 primarily upon the fitness of the starting genotype, and also upon the starting genotype itself (i.e., from which 376 location a population begins to explore an adaptive landscape) ${ }^{129,135}$.

378 An important limitation of this method is that it does not allow the detailed mapping of adaptive landscape 379 topography, because evolving populations typically harbour a large number of mutations whose contributions 380 to fitness are not easily disentangled ${ }^{136,137}$. Such a mapping requires more targeted approaches. One such 
381 approach is to engineer all possible genotypes in a small region of a landscape, for example by using all

382 combinations of the presence or absence of mutations that occurred along an adaptive evolutionary pathway, 383 or more comprehensively by using all possible combinations of mutations at a fixed number of nucleotide or 384 amino acid sites ${ }^{109}$ (Fig. 3e). One pertinent recent study constructed an adaptive landscape from all possible 385 combinations of 13 amino-acid-changing mutations at six amino acids in the heat-shock protein Hsp90 of $S$. 386 cerevisiae in a high-salt environment ${ }^{138}$. The resulting landscape provides several fundamental insights into 387 the evolvability of Hsp90 in this challenging environment. First, the landscape is dominated by epistasis: not 388 a single pairwise interaction between mutations is additive. These epistatic interactions include both positive 389 and negative epistasis, as well as sign epistasis. Second, the sign epistatic interactions produce landscape 390 ruggedness, with five local peaks and a single global peak that conveys a $10 \%$ increase in yeast growth rate 391 on high salt, relative to the wild-type genotype. Third, although the landscape is moderately rugged, it is still 392 highly navigable, as shown by simulated adaptive walks [G]. These walks reveal that the global peak can be 393 reached from nearly any starting point in the landscape. One important exception is the wild-type genotype, 394 because adaptive walks starting from this genotype tend to converge to a local peak but not to the global 395 peak. Taken together, these observations show how epistasis can generate landscape ruggedness, and that a 396 population's location within such a rugged landscape affects the ability of mutation to bring forth heritable, 397 adaptive phenotypic variation.

399 Another approach to constructing adaptive landscapes is based on deep mutational scanning ${ }^{139}$, in which 400 phenotypes are assayed for a large number of mutational variants of a single, typically wild-type genotype 401 (Fig. 3f). This approach thus characterizes the immediate neighbourhood of an adaptive peak. It has been 402 used extensively in recent years, for phenotypes as different as the 'splicing-in' of an exon ${ }^{116}$, the binding 403 affinity $^{114,115}$ and enzymatic activity ${ }^{12,113}$ of a protein, as well as the fitness of an entire organism ${ }^{84,110,111}$. For 404 example, a recent study employed a deep mutational scan of the wild-type sequence of the green fluorescent 405 protein from the jellyfish Aequorea victoria, using fluorescence level to define the landscape's surface ${ }^{140}$. 406 This analysis revealed a single, narrow peak centred on the wild-type sequence, with three quarters of the 
407 single-mutant sequences displaying reduced fluorescence, and half of the sequences with four mutations

408 showing no fluorescence at all. The analysis also revealed abundant negative epistasis, and very little positive 409 epistasis. Negative epistasis produces concave peaks ${ }^{141}$ (Fig. 3c), which reduces evolvability when a

410 population approaches an adaptive peak, because the amount of adaptive phenotypic variation accessible via

411 mutation decreases. Conversely, positive epistasis helps create convex peaks and facilitates evolvability.

412 These modes of epistasis also have implications for mutational robustness ${ }^{141,142}$. The concave peaks formed

413 by negative epistasis confer robustness, because individual mutations to genotypes on such peaks have small

414 fitness effects. By contrast, the convex peaks formed by positive epistasis confer sensitivity to mutation,

415 because individual mutations to genotypes on such peaks have large fitness effects. With few

416 exceptions ${ }^{143,144}$, a bias towards negative epistasis is among the most commonly reported features of

417 experimentally characterized adaptive landscapes ${ }^{110,111,114,115,138,140,141}$, in agreement with the diminishing

418 returns epistasis regularly observed in laboratory evolution experiments ${ }^{119,120,130-132}$.

420 Even though deep-mutational scanning and related techniques are powerful, they still render a typical

421 genotype space sparsely sampled, and extrapolating insights from the resulting incomplete landscapes to

422 complete landscapes is challenging ${ }^{138,145,146}$. Not affected by this limitation are small genotype spaces, where

423 it is possible to assay the phenotypes of all possible genotypes ${ }^{147,148}$ (Fig. $3 \mathrm{~g}$ ). One such genotype space is that

424 of short transcription factor binding sites, where one can measure how strongly a transcription factor binds to

425 thousands of different DNA sequences ${ }^{93}$. Such information is not just available for one, but for thousands of

426 transcription factors from multiple species ${ }^{149}$. Binding strength is an important molecular phenotype, because

427 it is a proxy for a factor's ability to activate or repress a target gene, and the gene expression patterns that

428 emerge from such binding events embody fundamental biological processes, including those in development,

429 physiology, and behaviour. Importantly, the location and timing of these gene expression patterns can be

430 fine-tuned, or altogether transformed, by mutations that affect the strength of transcription factor-DNA

431 interactions ${ }^{150,151}$. The mapping of DNA sequence to binding strength can therefore be thought of as an 
432 adaptive landscape, in which mutation and natural selection optimize the capacity of a DNA sequence to bind 433 a transcription factor.

434

435 A recent study analyzed the topographies of more than 1000 such landscapes ${ }^{94}$. They contained little sign 436 epistasis, and therefore typically comprised only a single peak. Similar to the landscape of yeast Hsp90 in 437 high salinity ${ }^{138}$, these landscapes were highly navigable. Their global peaks tended to be accessible from 438 throughout the landscape via a series of 'uphill' mutational steps. Indeed, even at the furthest mutational 439 distance from a global peak, more than $20 \%$ of all possible mutational paths were accessible. Such smooth 440 landscapes facilitate evolvability, because mutation can readily bring forth beneficial phenotypic variation, 441 regardless of a population's location on the landscape.

443 A limitation to these approaches, as compared to experimental evolution, is that an adaptive landscape for a 444 single binding site or an individual gene has many fewer dimensions than an adaptive landscape for an entire 445 genome. This is important, because the valleys that separate adaptive peaks in low-dimensional landscapes 446 may not do so in high-dimensional landscapes. The reason is that increased dimensionality may create 447 mutational paths that bridge adaptive valleys, or that transform local adaptive peaks into saddle points [G].

448 Such extra-dimensional bypasses [G] increase the accessibility of adaptive peaks, and thus increase

449 evolvability ${ }^{5}$. Long the subject of theoretical research ${ }^{5,152}$, extra-dimensional bypasses have recently been 450 uncovered in an adaptive landscape of binding affinity for the protein GB1 of Streptococcal bacteria ${ }^{153}$. The 451 authors analyzed all $20^{4}$ protein variants of 4 amino acid sites, and sampled $\sim 20,000$ pairs of mutations that 452 exhibited reciprocal sign epistasis (Fig. 3d). Of these pairs, $\sim 15 \%$ exhibited an extra-dimensional bypass 453 when one of the other two amino acid sites was considered. Such an increase in the mutational accessibility 454 of adaptive peaks suggests that increasing the dimensionality of adaptive landscapes from that of individual 455 binding sites or genes to that of entire genomes reduces landscape ruggedness and thus enhances evolvability. 
457 The examples highlighted here are only a small sample of recent experimental studies of adaptive landscapes, 458 with other pertinent examples in systems as different as drug delivery vehicles ${ }^{154}$ and cancer ${ }^{155}$. We anticipate 459 that the resolution and scale of such landscapes will continue to increase as high-throughput genotyping and 460 phenotyping technologies advance (Box 1).

461

\section{2 [H1] Evolvability evolving}

463 Any cause or mechanism of evolvability could in principle itself be subject to evolutionary change. Three 464 questions about such change are germane. First, can the mechanism evolve in principle, i.e., is there genetic 465 variation in it? Second, does it evolve, either in nature or in the laboratory? Third, is a change in evolvability 466 itself adaptive? Or is it instead a by-product of other adaptations or of non-adaptive processes, such as 467 developmental constraints, mutation bias, or genetic drift? We discuss existing evidence pertaining to these 468 questions for each of our three major causes of evolvability.

469

[H2] Evolution of phenotypic heterogeneity. Genetic mechanisms that create phenotypic heterogeneity can 471 evolve. For example, the rate of DNA mutation is itself subject to evolutionary change ${ }^{156,157}$, because the 472 DNA repair enzymes that keep DNA mutations in check can themselves undergo mutations that lead to 473 elevated mutation rates. Such evolution can be adaptive in novel environments ${ }^{156,158}$, for example during 474 E.coli's colonization of the mouse gut ${ }^{159}$. Similarly, increases in recombination rate can accelerate a 475 population's rate of adaptation — a proxy for evolvability — either by creating more beneficial allele 476 combinations or by helping to eliminate deleterious mutations ${ }^{160}$.

478 Non-genetic mechanisms of phenotypic heterogeneity can also evolve ${ }^{161}$. For example, gene expression noise 479 levels vary genetically with promoter strength and with the strength of transcription factor binding sites $^{162}$; 480 stop-codon readthrough rates vary with stop-codon identity (UAG, UAA or UGA), the surrounding sequence 481 context, and the structure of $\mathrm{mRNA}^{163}$; the formation and activity of prions varies according to the presence 482 of aggregation-prone amino acid sequences in prion-forming protein domains, such as glutamine/asparagine- 
483 rich sequences ${ }^{164}$; and protein promiscuity varies with a protein's coding sequence ${ }^{61,67,105}$. Thus, in each case, 484 the factors that can affect phenotypic heterogeneity are genetically encoded, and can therefore evolve.

486 What is more, mechanisms that create phenotypic heterogeneity do evolve, both in laboratory experiments 487 and in nature. For example, the evolution of increased gene expression noise in S. cerevisiae has been 488 reported for antifungal resistance genes in the $1 a b^{34}$ and for plasma-membrane transporters in the wild ${ }^{165}$. 489 Experimental evolution of synthetic E. coli promoters to specific mean expression levels results in promoters 490 with low expression noise, suggesting that the noisy expression of many natural E. coli promoters is an 491 evolved property ${ }^{166}$. Other forms of phenotypic heterogeneity have also been successfully evolved in the lab, 492 including protein promiscuity in bacteriophage $\lambda\left(\mathrm{ref}^{67}\right.$ ) and the stochastic switching of colony morphology 493 in Pseudomonas fluorescens ${ }^{17}$.

495 At least in some instances, the evolvability conferred by phenotypic heterogeneity may have evolved because 496 it was adaptive. For example, in the experimental evolution of populations of S. cerevisiae exposed to 497 antifungal stress, increased expression noise evolved in the synthetic regulatory circuits controlling an 498 antifungal resistance gene, because it enhanced the adaptive value of beneficial mutations ${ }^{34}$. Similarly, in the 499 experimental evolution of populations of $P$. fluorescens exposed to environmental fluctuations, the stochastic 500 switching of colony morphology evolved as an adaptive bet-hedging strategy ${ }^{17}$. Such a strategy was also 501 observed in the experimental evolution of $E$. coli under antibiotic stress, where the stochastic expression of 502 persister cells evolved to facilitate survival in high concentration of antibiotic ${ }^{26}$. In other instances, 503 evolvability is a by-product of other adaptations. For example, promiscuity in the host-recognition protein of 504 bacteriophage $\lambda$ evolved as a by-product of selection for increased absorption to the virus' native cell surface 505 receptor $^{67}$. Specifically, the same mutations that increased absorption also destabilized the protein, producing $506 \lambda$ particles that were proficient at targeting different receptors. 
508 [H2] Evolution of robustness. Variation in mutational robustness is found at all scales of biological 509 organization, including the structures of macromolecules ${ }^{71,147}$, interactions between macromolecules and their 510 ligands ${ }^{87,92}$, as well as the gene expression patterns of regulatory circuits ${ }^{167}$. Mutational robustness can 511 therefore evolve. Moreover, it can evolve by various means; for example, via increased protein stability ${ }^{71}$ or $^{2}$ 512 via gene duplication ${ }^{74}$.

\section{3}

514 Mutational robustness also has evolved, both in nature and in the laboratory. For example, the structures of 515 eukaryotic microRNA precursor stem-loops are more robust to mutation than random RNA sequences with 516 similar stem-loop structures ${ }^{168}$, and the mutational robustness of a protein's tertiary structure tends to increase 517 with the protein's age ${ }^{169}$. Directed protein evolution has demonstrated that mutational robustness of 518 cytochrome P450 proteins can increase in sufficiently large populations ${ }^{170}$, and experimental evolution of $S$. 519 cerevisiae has demonstrated that gene duplications can confer mutational robustness ${ }^{74}$.

521 We are not aware of experimental evidence that mutational robustness has evolved because it causes 522 evolvability. By contrast, there is evidence that mutational robustness has evolved because it is itself 523 adaptive ${ }^{171}$, for example in viral populations exposed to chemical mutagens, because robustness provides a 524 competitive advantage when the mutation rate is elevated ${ }^{172}$. In addition, mutational robustness may often 525 evolve as a by-product of other adaptations. For example, chaperones help maintain proteome integrity 526 during environmental stress, and may buffer mutations only as a side effect. Similarly, the mutational 527 robustness of eukaryotic microRNA precursor stem-loops is likely to be a by-product of selection for 528 robustness of these RNA structures to temperature fluctuations ${ }^{173}$.

530 [H2] Evolution of adaptive landscape topography. This cause of evolvability can also evolve: the location of 531 an individual or a population on an adaptive landscape can change through DNA mutations or recombination, 532 and because local landscape topography may differ in different locations, so may evolvability ${ }^{91,135,138,141,147,174-}$ $533{ }^{176}$. A comparison of the fitness effects of mutations to three orthologous TIM barrel proteins provides an 
534 illustrative example ${ }^{175}$. These proteins are distantly related, retaining only $\sim 30-40 \%$ sequence identity, but 535 they have the same fold and function. They therefore occupy different locations on the same adaptive 536 landscape. These locations differ in their evolvability, because the same mutations have different, albeit 537 correlated fitness effects in the three sequence backgrounds (locations). Another example is provided by the 538 experimental evolution of two divergent yeast strains in the same laboratory conditions ${ }^{129}$. These strains, 539 which differ at roughly 50,000 single nucleotide sites and therefore occupy different locations on their 540 adaptive landscape, also differ in the rate at which they adapt evolutionarily — a proxy for evolvability ${ }^{129,177}$.

541 Analysis of quantitative trait loci [G] partly attributes this difference in evolvability to a small subset of 542 mutations, such as those involved in the ribosome biogenesis pathway.

543

544 The evolvability conferred by a landscape's local topography has also evolved. As shown in Fig. 2, for 545 example, eleven substitutions occurred during the evolution of an ancient steroid hormone receptor, and this 546 change in adaptive landscape location dramatically altered the spectrum of DNA-binding phenotypes 547 accessible via mutation ${ }^{91}$. An additional example comes from Lenski's long-term ( $>60,000$ generations) 548 evolution experiment with E. coli populations ${ }^{178}$. Here, one out of twelve populations evolved the ability to 549 utilize citrate, and did so after 31,500 generations. The mutation needed to evolve citrate utilization conferred 550 a fitness benefit even in the original ancestor of the experiment, but other mutations that occurred during the 551 initial stages of the experiment conferred larger fitness benefits, and created a genetic background in which 552 the initial citrate utilization-mutation no longer conferred a fitness benefit. Thus, evolution drove the 553 population to a location on the adaptive landscape that precluded the evolution of citrate utilization. Only 554 later did subsequent mutations bring the population back to a location where this mutation was adaptive.

556 The same experiment also provides further evidence for evolving evolvability ${ }^{177}$. Within the first 500 557 generations of this experiment, multiple genetically distinct subpopulations had evolved within a single 558 population, meaning that the population had diversified from the location of the ancestral genotype to 559 multiple new locations on the adaptive landscape. One of these subpopulations would eventually outcompete 
560 the others, but it was not the subpopulation with the highest fitness. Rather, it was a subpopulation located in 561 a region of the adaptive landscape that had higher evolvability. This was shown by 'replay experiments', in 562 which 10 replicate populations were evolved from distinct founding subpopulations — that is, from distinct 563 locations on the adaptive landscape. The subpopulation that would eventually outcompete the others 564 generated more beneficial phenotypic variation than the other subpopulations — it had higher evolvability. 565 After $\sim 900$ generations of evolution from these distinct landscape locations, the subpopulations evolved from 566 the high-evolvability location tended to outcompete those evolved from other locations.

567

568 We are not aware of experimental evidence that a population's location on an adaptive landscape has evolved 569 because it conferred evolvability. For instance, in the preceding example, evolvability evolved as a by570 product of the fixation of neutral or beneficial mutations that just happened to drive one of the subpopulations 571 toward a high-evolvability region of the landscape ${ }^{177}$. Non-adaptive forces may also explain the evolution of 572 a population's location on an adaptive landscape. For example, the eleven substitutions that occurred during 573 the evolution of an ancient steroid hormone receptor did not alter the protein's binding specificity, which 574 suggests that genetic drift caused this change in landscape location and the corresponding dramatic shift in 575 evolvability ${ }^{90}$. An alternative possibility is that this change in landscape location was due to selection for 576 protein function unrelated to binding specificity.

577

578 Taken together, these examples show that the three causes of evolvability highlighted here — phenotypic 579 heterogeneity, robustness, and adaptive landscapes — are themselves subject to evolutionary change. 580 Whether they often evolve because they confer evolvability remains a particularly challenging open question.

\section{2 [H1] Outlook}

583 Driven by technological advances, research into all three causes of evolvability is progressing in leaps and 584 bounds. We anticipate that this progress is going to continue unabated. For example, the currently well585 studied mechanisms to create non-genetic phenotypic heterogeneity that we discuss may well be only a small 
586 subset of all pertinent mechanisms. Future work may reveal others to be important as well, such as RNA 587 editing ${ }^{179}$ and protein allostery ${ }^{180}$. In addition, we know little about how conflicts of selection may influence 588 the evolution of such mechanisms, especially in organisms that are not clonally related (Box 2). As for 589 robustness, we understand its causes well for some systems like proteins or duplicate genes, but much less 590 well for systems of greater complexity, such as gene regulatory circuits and metabolism. The evolutionary 591 consequences of robustness become amply clear from detailed reconstructions of the evolution of molecules 592 such as steroid hormone receptors ${ }^{91}$, but to date few such reconstructions are available. In the context of 593 adaptive landscapes, we are only beginning to understand how landscape topography depends on higher594 order epistasis ${ }^{181,182}$. Moreover, although we know that the environment can affect adaptive landscape 595 topography, we know little about how it does ${ }^{86,183}$. We are also only beginning to understand how our 596 knowledge of landscape topography may facilitate the prediction of evolutionary trajectories ${ }^{109,184}$, or the 597 deliberate redirection of evolving populations of pathogens toward low-evolvability regions of a landscape ${ }^{185}$.

599 The three major causes of evolvability interact, but we do not fully understand how or to what effect. For 600 example, phenotypic heterogeneity can smoothen an adaptive landscape, if a genotype's overall fitness is 601 equal to the average fitness of each of the phenotypes it brings forth ${ }^{33}$. Similarly, a DNA mutation that 602 renders a protein's phenotype robust to further mutations can be viewed as displacing the genotype to a 603 smooth region of an adaptive landscape, where further mutations have smaller phenotypic effects. However, 604 the degree of such 'smoothing' has not been explicitly characterized for any experimentally studied 605 landscape. When an organism generates non-genetic adaptive variation in phenotypes, it creates two or more 606 phenotypes from the same genotype, but any one adaptive phenotype can be stabilized by DNA mutations 607 only if the starting genotype resides in a region of an adaptive landscape where some of its mutants provide 608 such stabilization. We do not know the extent to which non-genetic mechanisms that create phenotypic 609 variation and increase evolvability ensure that the variation they cause can be genetically stabilized. Finally, 610 because a phenotype's robustness to genetic and non-genetic change are often correlated ${ }^{69}$, genotypes that are 611 especially robust to DNA mutations may also bring forth less phenotypic heterogeneity by non-genetic 
612 means. If so, trade-offs between robustness and non-genetic mechanisms to create phenotypic heterogeneity

613 may exist, and these trade-offs are well-worth exploring.

614

615 A final frontier regards the evolution of the various evolvability mechanisms themselves. As we have shown,

616 there is ample evidence that all three mechanisms can and do change in biological evolution. However, we

617 have less information about whether their existence reflects an adaptive value of evolvability. Does increased

618 mutational robustness at least sometimes come about because it enhances evolvability? Has the ruggedness of

619 some adaptive landscapes decreased in the course of evolution, and if so, is it because reduced ruggedness

620 increases evolvability? Questions like these are fascinating and profound, because an affirmative answer

621 means that life itself can help create the conditions that ensure its advancement.

622

\section{Box $1 \mid$ Methodological advances}

624 Our ability to study the molecular causes of evolvability has been greatly improved by recent methodological

625 advances. For example, our growing understanding of phenotypic heterogeneity is driven by microfluidic

626 devices and time-lapse microscopy, which provide information about the compositions, morphologies and

627 growth rates of single cells in dynamic environments ${ }^{186}$. Complementary information is provided by methods

628 such as fluorescence in situ hybridization and single-cell RNA-seq, which describe the location and

629 abundance of mRNA transcripts, respectively ${ }^{187,188}$. Combined with whole-genome sequencing, such methods

630 have detailed the molecular causes of phenotypic heterogeneity, such as how stochastic gene expression

631 drives persistence in bacteria ${ }^{26}$ and rare-cell variability in cancer ${ }^{24}$. Non-single-cell methodologies have also

632 furthered our understanding of phenotypic heterogeneity. For example, ribosome footprint profiling, which

633 characterizes the distribution of ribosomes on mRNA transcripts ${ }^{189}$, has detailed the prevalence of stop-codon

634 readthrough in yeast, fly, and human ${ }^{39}$.

636 Several methodological advances have improved our understanding of mutational robustness and of adaptive 637 landscapes. For example, approaches that characterize a small region of an adaptive landscape typically rely 
638 on deep mutational scanning ${ }^{139}$, a method that combines systematic mutagenesis with high-throughput

639 phenotypic assays. These assays include fluorescence-activated cell sorting, which can be used to measure

640 protein functions such as fluorescence or ligand binding, as well as EMPIRIC ${ }^{190}$, which can measure the

641 fitness of many cells in parallel. To capture the effects of mutations in their native genomic context, genome-

642 editing tools such as CRISPR-Cas9 can be used to introduce mutations to specific chromosomal $\operatorname{loci}^{103}$.

643 Approaches that exhaustively characterize an entire (small) genotype space have profited from chip-based

644 technologies that simultaneously assay the phenotypes of all possible genotypes ${ }^{93}$, as well as from high-

645 throughput in vitro selection methodologies that systematically enrich an initially random library of

646 sequences for those sequences that perform a particular function, such as binding a ligand ${ }^{147}$.

647 To understand how these causes of evolvability have changed over long evolutionary timescales, they are

648 often combined with maximum likelihood methods to statistically infer and experimentally reconstruct the

649 genotypes and phenotypes of ancient macromolecules ${ }^{191}$.

650

651 Box 2 | Conflicts between different levels of selection

652 Biological systems are hierarchically organized, with macromolecules embedded in cells, cells in whole 653 organisms, and organisms in populations. A genetic change that is beneficial on one level of this hierarchy 654 may be detrimental on another. For example, because most random DNA mutations have detrimental effects 655 on individuals or their offspring ${ }^{192}$, DNA mutations that increase the DNA mutation rate itself will also be 656 detrimental for most individuals. By contrast, they may be advantageous for a population as a whole, 657 especially in a stressful environment, where a few beneficial mutant individuals may ensure survival ${ }^{158,193}$ or 658 accelerate adaptation ${ }^{156}$. Such conflicts are also relevant for the evolvability mechanisms we discuss, such as 659 those that generate non-genetic heterogeneity, because in most environments such heterogeneity will not 660 benefit all individuals ${ }^{15,22,25}$. Various approaches help predict how evolution can resolve such conflicts $^{194-198 .}$. 661 Among them are multi-level selection theory ${ }^{197}$ and kin selection theory ${ }^{196}$. The latter shows that higher, 662 population-level adaptations can evolve and persist whenever populations consist of genetically highly 663 related individuals, because in this case, the genetic 'interests' of individuals are aligned with those of the 
664 population. It is relevant here that many known cases of adaptive non-genetic heterogeneity are found in 665 clonal populations of genetically identical individuals ${ }^{15}$, where an individual's interests are served as long as 666 some of its clone-mates survive. Although theoretical work shows that evolvability mediated by prions such 667 as $\left[\mathrm{PSI}^{+}\right]$may persist in non-clonal populations of the yeast Saccharomyces cerevisae ${ }^{85,199}$, extending such 668 insights to other mechanisms of phenotypic heterogeneity, particularly non-heritable mechanisms, and to a 669 broader range of organisms remains an important task for future work.

670

671 With respect to robustness, the dual property to phenotypic heterogeneity, we note that it is often 672 advantageous to an individual, for example when a mutation creates a thermodynamically more stable protein 673 that is less prone to misfolding or inactivation ${ }^{170}$. Wherever this is the case, the individual-level advantage 674 and the population-level advantage of evolvability are aligned. This makes robustness a cause of evolvability 675 whose evolutionary origin need not involve conflict, and is thus especially easy to explain. At the same time, 676 this absence of conflict also means that it is more difficult to disentangle whether the robustness of any one 677 trait originated in an individual-level advantage, such as the robustness that chaperones provide to 678 proteomes $^{200}$, or in a 'second-order' advantage of evolvability, which chaperones also provide ${ }^{82}$.

679

680 Figure legends

681 Figure 1 | Phenotypic heterogeneity is a cause of evolvability. a | Phenotypic heterogeneity can generate

682 a small subpopulation of cells that exhibits a new phenotype, such as a persister phenotype (red cells in

683 environment 1). Such a phenotype can be adaptive, because it allows a subpopulation to survive an

684 environmental challenge, such as antibiotic exposure (environment 2). Mutation (red cross) may stabilize the

685 phenotype, or it may generate a different phenotype that is adaptive in the new environment, such as a

686 mutation that confers resistance to an already tolerant bacterial cell. There are many sources of phenotypic

687 heterogeneity: b | Stochastic gene expression causes mRNA transcript levels to vary among cells. C | Errors

688 in protein synthesis, such as mistranslation, cause variation in the amino acid sequences of proteins that are 
translated from the same mRNA transcript. d | Epigenetic modifications, such as the yeast prion $[P S I+]$,

690 cause variation in protein sequences, in this example via stop-codon readthrough.

691

692 Figure 2 I Robustness causes evolvability by providing access to a diversity of mutational

693 neighbourhoods. a,b $\mid$ The mutational neighbourhoods of the ancestral steroid receptor (AncSR1 in ref ${ }^{91}$;

694 part a) and the derived steroid receptor after 11 amino acid changes (AncSR1+11p in ref ${ }^{91}$; part b). Each

695 vertex (circle) corresponds to a sequence of amino acids at four sites in each protein's recognition helix: the

696 three that historically changed binding specificity, plus an adjacent site. Of all 160,000 possible such

697 sequences in each background, only functional sequences are shown - i.e., sequences that bind the oestrogen

698 (pink) or the steroid (blue) response elements, or that promiscuously bind both (yellow). Edges connect

699 sequences that differ in a single amino acid. The number of functional sequences differs dramatically

700 between the two backgrounds: 129 in the ancestral background, as compared to 1,351 in the derived

701 background. $\mathbf{c}, \mathbf{d} \mid$ Moreover, the lengths of the shortest paths from a sequence that binds the oestrogen

702 response element to a sequence that binds the steroid response element is much longer in the ancestral

703 background (part c) than in the derived background (part d). The * symbol indicates starting points from

704 which there is no path to a sequence that binds the steroid response element. Data from ref ${ }^{91}$. [Copy Ed: no

705 credit line is needed for actual figure adaptation. Although the data are derived from Ref91, the figures

706 themselves are not from there (or even from the supp info of the original article). It's also Nature

707 anyway, so no formal copyright clearance would be needed anyway.]

709 Figure 3 | Adaptive landscape topography influences evolvability. a | A smooth, single-peaked

710 landscape facilitates evolvability, because mutations can create adaptive phenotypic variation from anywhere

711 in the landscape, except atop the global peak. For example, the white and black circles denote two distinct

712 mutational paths that start from different points in the landscape, but that both converge on the global peak

713 via a series of 'uphill' mutational steps. b | By contrast, a multi-peaked, or rugged landscape hinders

714 evolvability, because an evolving population may become trapped on local, suboptimal peaks. For example, 
715 whereas the mutational path indicated by the white circles leads to the global peak, the mutational path

716 indicated by the black circles does not. c | The shape of an adaptive peak is a consequence of magnitude

717 epistasis. Specifically, positive epistasis generates peaks that are convex, whereas negative epistasis generates

718 peaks that are concave. As a population climbs an adaptive peak, evolvability tends to increase if the peak is

719 convex, whereas it tends to decrease if the peak is concave. $\mathbf{d}$ | Landscape ruggedness is a consequence of

720 sign epistasis, which creates adaptive valleys that may be difficult for an evolving population to cross. Grey

721 circles correspond to those in part b. e-g | The same landscape as in part a, but shown as two-dimensional

722 contour plots. Open circles indicate genotypes and edges connect genotypes that differ by a single mutation.

723 The same landscape can be studied by: systematically engineering genotypes that contain all possible

724 combinations of a small number of mutations (part e); deep mutational scanning of a single wild-type

725 genotype, including all single-mutants, many double-mutants, and some triple-mutants (part f); or in the case

726 of small landscapes, via the exhaustive enumeration of all possible genotypes (part $\mathbf{g}$ ).

727

\section{Glossary}

729 Isogenic populations

730 Populations of individuals with the same genotype.

731

732 Phenotypic plasticity

733 The ability of one genotype to produce more than one phenotype in response to different environmental stimuli.

\section{Modularity}

736 The extent to which a system can be partitioned into distinct components.

738 Pleiotropy

739 When one gene or one mutation affects multiple phenotypes.

740

741 Pre-mutation evolvability 
742 Evolvability driven by new mutations.

743

744 Post-mutation evolvability

745 Evolvability driven by existing genetic variation within a population, for example via recombination acting on that

746 variation.

747

748 Gene expression noise

749 Variability among isogenic cells in transcript or protein abundance.

750

$751 \quad$ Viral latency

752 The ability of a virus to remain dormant in a host cell.

753

754 Competence

755 The ability of a cell to take up DNA from the environment.

756

757 Tolerance

758 The ability of bacteria to survive in the presence of antibiotics without developing resistance.

759

760 Population bottleneck

761 A temporary, drastic reduction in population size.

762

763 Genetic assimilation

764 A process by which a new phenotype that results from an environmental perturbation becomes genetically encoded.

765

$766 \quad$ Kinetic trapping

767 Occurs when a protein does not reach its minimum free-energy structure, but rather becomes trapped in a non-

768 equilibrium structure.

769

770 Stop-codon readthrough 
771 When translation does not terminate at a stop codon, but rather continues to extend an amino acid chain.

772

773 Prions

774 Proteins that propagate by inducing properly folded proteins to convert into a misfolded form, often resulting in

775 aggregation.

776

777 Cryptic genetic variation

778 Genetic variation that normally causes little to no phenotypic variation, but that has the potential to cause phenotypic

779 variation in new environments or new genetic backgrounds.

780

781 Enhancer

782 A short DNA sequence that is bound by regulatory proteins to activate the transcription of a gene, which may be located 783 many thousands of base pairs away.

784

785 Chaperones

786 Proteins that assist other proteins in folding, or refold misfolded proteins.

787

788 Epistatic interactions

789 Non-additive interactions between alleles in their contribution to a phenotype or fitness.

790

791 Protein domain

792 A distinct functional and often autonomously folding unit of a protein.

793

794 Genotype space

795 The space of all possible genotypes. For a nucleic acid sequence of length $L$, this space comprises $4^{L}$ genotypes.

796

797 Concave

798 A real-valued function on an interval of real numbers is concave if any line connecting two points on the graph of the 799 function lies on or below the graph. 
801 Convex

802 A real-valued function on an interval of real numbers is convex if any line connecting two points on the graph of the 803 function lies above or on the graph.

804

805 Adaptive walks

806 A series of mutations that never decrease fitness.

807

808 Saddle points

809 Points on a landscape that have zero slope in at least two orthogonal directions, yet are not local peaks.

810

811 Extra-dimensional bypasses

812 Accessible mutational paths to an adaptive peak that are faciltated by increasing the dimensionality of an adaptive

813 landscape.

814

815 Quantitative trait loci

816 Loci that explain part of the genetic basis of variation in a phenotype.

817

\section{Key points}

819 Evolvability is the ability of a biological system to produce phenotypic variation that is both heritable $820 \quad$ and adaptive.

821

- Recent technological advances are transforming evolvability research from a field dominated by theory to one illuminated by experiment.

- We highlight three causes of evolvability that have been the focus of recent experimental research. They are phenotypic heterogeneity, robustness, and adaptive landscape topography.

825 - We discuss the mounting evidence that these causes of evolvability can evolve, and also the question 826 of whether they can evolve adaptively. 


\section{Acknowledgements}

829 We thank Martin Ackermann, Balazs Bogos, Steven A. Frank, Jordi Van Gestel, Alex R. Hall, Daan Kiviet,

$830 \&$ Macarena Toll Riera for discussions, and the reviewers for their constructive criticism. We apologize to

831 our colleagues whose important contributions to evolvability research could not be covered due to space

832 constraints. J.L.P. acknowledges support from Swiss National Science Foundation Grant PP00P3_170604.

833 A.W. acknowledges support from the European Research Council Advanced Grant 739874, Swiss National

834 Science Foundation Grant 31003A_1728887, and the University Priority Research Program in Evolutionary

835 Biology at the University of Zurich. J.L.P. and A.W. are also affiliated with the Swiss Institute of

836 Bioinformatics and A.W. is also affiliated with the Santa Fe Institute.

837

838 Author contributions

839 J.L.P. and A.W. contributed equally to all aspects of this work: researching and discussing content, writing

840 the manuscript and reviewing/editing it before submission.

841

\section{Reviewer information}

843 Nature Reviews Genetics thanks G. Wagner, J. Zhang and the other, anonymous

844 reviewer(s) for their contribution to the peer review of this work.

1 Dawkins, R. in Artificial Life: The Proceedings of an Interdisciplinary Workshop on the Synthesis and Simulation of Living Systems. (ed C. G. Langton) 201-220 (Addison-Wesley, Reading, MA) (1989).

An influential discussion of evolvability and its evolution, set in the context of artificial embryological systems. 
$2 \quad$ Nuno de la Rosa, L. Computing the Extended Synthesis: Mapping the Dynamics and Conceptual Structure of the Evolvability Research Front. J Exp Zool B Mol Dev Evol 328, 395-411, doi:10.1002/jez.b.22741 (2017).

3 Pigliucci, M. Is evolvability evolvable? Nat Rev Genet 9, 75-82, doi:10.1038/nrg2278 (2008).

$4 \quad$ Alberch, P. From genes to phenotype: dynamical systems and evolvability. Genetica 84, 511 (1991).

5 Conrad, M. The geometry of evolution. Biosystems 24, 61-81 (1990).

6 Hansen, T. F. Is modularity necessary for evolvability? Remarks on the relationship between pleiotropy and evolvability. Biosystems 69, 83-94 (2003).

$7 \quad$ Houle, D. Comparing evolvability and variability of quantitative traits. Genetics 130, 195204 (1992).

8 Kauffman, S. A. Requirements for Evolvability in Complex-Systems - Orderly Dynamics and Frozen Components. Physica D 42, 135-152, doi:Doi 10.1016/0167-2789(90)90071-V (1990).

9 Kirschner, M. \& Gerhart, J. Evolvability. Proc Natl Acad Sci U S A 95, 8420-8427 (1998).

10 Wagner, A. Does Evolutionary Plasticity Evolve? Evolution 50, 1008-1023, doi:10.1111/j.1558-5646.1996.tb02342.x (1996).

11 Wagner, G. P. \& Altenberg, L. Perspective: Complex Adaptations and the Evolution of Evolvability. Evolution 50, 967-976, doi:10.1111/j.1558-5646.1996.tb02339.x (1996).

12 Diaz Arenas, C. \& Cooper, T. F. Mechanisms and selection of evolvability: experimental evidence. FEMS Microbiol Rev 37, 572-582, doi:10.1111/1574-6976.12008 (2013).

13 Masel, J. \& Trotter, M. V. Robustness and evolvability. Trends Genet 26, 406-414, doi:10.1016/j.tig.2010.06.002 (2010).

14 Galhardo, R. S., Hastings, P. J. \& Rosenberg, S. M. Mutation as a stress response and the regulation of evolvability. Crit Rev Biochem Mol Biol 42, 399-435, doi:10.1080/10409230701648502 (2007).

15 Ackermann, M. A functional perspective on phenotypic heterogeneity in microorganisms. Nat Rev Microbiol 13, 497-508, doi:10.1038/nrmicro3491 (2015).

16 Tawfik, D. S. Messy biology and the origins of evolutionary innovations. Nat Chem Biol 6, 692-696, doi:10.1038/nchembio.441 (2010).

17 Beaumont, H. J., Gallie, J., Kost, C., Ferguson, G. C. \& Rainey, P. B. Experimental evolution of bet hedging. Nature 462, 90-93, doi:10.1038/nature08504 (2009).

18 True, H. L. \& Lindquist, S. L. A yeast prion provides a mechanism for genetic variation and phenotypic diversity. Nature 407, 477-483 (2000).

19 Blake, W. J., M, K. A., Cantor, C. R. \& Collins, J. J. Noise in eukaryotic gene expression. Nature 422, 633-637, doi:10.1038/nature01546 (2003).

20 Ozbudak, E. M., Thattai, M., Kurtser, I., Grossman, A. D. \& van Oudenaarden, A. Regulation of noise in the expression of a single gene. Nat Genet 31, 69-73, doi:10.1038/ng869 (2002).

21 Elowitz, M. B., Levine, A. J., Siggia, E. D. \& Swain, P. S. Stochastic gene expression in a single cell. Science 297, 1183-1186, doi:10.1126/science.1070919 (2002).

22 Raj, A. \& van Oudenaarden, A. Nature, nurture, or chance: stochastic gene expression and its consequences. Cell 135, 216-226, doi:10.1016/j.cell.2008.09.050 (2008).

23 Sanchez-Romero, M. A. \& Casadesus, J. Contribution of phenotypic heterogeneity to adaptive antibiotic resistance. Proc Natl Acad Sci U S A 111, 355-360, doi:10.1073/pnas.1316084111 (2014).

24 Shaffer, S. M. et al. Rare cell variability and drug-induced reprogramming as a mode of cancer drug resistance. Nature 546, 431-435, doi:10.1038/nature22794 (2017).

25 Harms, A., Maisonneuve, E. \& Gerdes, K. Mechanisms of bacterial persistence during stress and antibiotic exposure. Science 354, doi:10.1126/science.aaf4268 (2016). 
26 Levin-Reisman, I. et al. Antibiotic tolerance facilitates the evolution of resistance. Science 355, 826-830, doi:10.1126/science.aaj2191 (2017).

This study showed that tolerance against ampicillin facilitates the evolution of resistance.

27 Ramirez, M. et al. Diverse drug-resistance mechanisms can emerge from drug-tolerant cancer persister cells. Nat Commun 7, 10690, doi:10.1038/ncomms10690 (2016).

28 Sharma, S. V. et al. A chromatin-mediated reversible drug-tolerant state in cancer cell subpopulations. Cell 141, 69-80, doi:10.1016/j.cell.2010.02.027 (2010).

29 Spencer, S. L., Gaudet, S., Albeck, J. G., Burke, J. M. \& Sorger, P. K. Non-genetic origins of cell-to-cell variability in TRAIL-induced apoptosis. Nature 459, 428-432, doi:10.1038/nature08012 (2009).

30 Frank, S. A. \& Rosner, M. R. Nonheritable cellular variability accelerates the evolutionary processes of cancer. PLOS Biol 10, e1001296, doi:10.1371/journal.pbio.1001296 (2012).

31 West-Eberhard, M. Developmental plasticity and evolution. (Oxford University Press, 2003).

32 Waddington, C. H. The genetic assimilation of an acquired character. Evolution 7, 118-126 (1953).

33 Frank, S. A. Natural selection. II. Developmental variability and evolutionary rate. J Evol Biol 24, 2310-2320, doi:10.1111/j.1420-9101.2011.02373.x (2011).

34 Bodi, Z. et al. Phenotypic heterogeneity promotes adaptive evolution. PLoS Biol 15, e2000644, doi:10.1371/journal.pbio.2000644 (2017).

35 Drummond, D. A. \& Wilke, C. O. The evolutionary consequences of erroneous protein synthesis. Nat Rev Genet 10, 715-724, doi:10.1038/nrg2662 (2009).

36 Goldsmith, M. \& Tawfik, D. S. Potential role of phenotypic mutations in the evolution of protein expression and stability. Proc Natl Acad Sci U S A 106, 6197-6202, doi:10.1073/pnas.0809506106 (2009).

37 Javid, B. et al. Mycobacterial mistranslation is necessary and sufficient for rifampicin phenotypic resistance. Proc Natl Acad Sci U S A 111, 1132-1137, doi:10.1073/pnas.1317580111 (2014).

38 Miranda, I. et al. Candida albicans CUG mistranslation is a mechanism to create cell surface variation. MBio 4, doi:10.1128/mBio.00285-13 (2013).

39 Dunn, J. G., Foo, C. K., Belletier, N. G., Gavis, E. R. \& Weissman, J. S. Ribosome profiling reveals pervasive and regulated stop codon readthrough in Drosophila melanogaster. Elife 2, e01179, doi:10.7554/eLife.01179 (2013).

40 Freitag, J., Ast, J. \& Bolker, M. Cryptic peroxisomal targeting via alternative splicing and stop codon read-through in fungi. Nature 485, 522-525, doi:10.1038/nature11051 (2012).

41 Jungreis, I. et al. Evolutionary Dynamics of Abundant Stop Codon Readthrough. Mol Biol Evol 33, 3108-3132, doi:10.1093/molbev/msw189 (2016).

42 Masel, J. Cryptic genetic variation is enriched for potential adaptations. Genetics 172, 19851991, doi:10.1534/genetics.105.051649 (2006).

43 Whitehead, D. J., Wilke, C. O., Vernazobres, D. \& Bornberg-Bauer, E. The look-ahead effect of phenotypic mutations. Biol Direct 3, 18, doi:10.1186/1745-6150-3-18 (2008).

44 Borenstein, E., Meilijson, I. \& Ruppin, E. The effect of phenotypic plasticity on evolution in multipeaked fitness landscapes. J Evol Biol 19, 1555-1570, doi:10.1111/j.14209101.2006.01125.x (2006).

45 Yanagida, H. et al. The Evolutionary Potential of Phenotypic Mutations. PLoS Genet 11, e1005445, doi:10.1371/journal.pgen.1005445 (2015).

This study demonstrated that in the natural history of $S$. cerevisiae, a phenotypic mutation was reinforced via gene duplication and mutation. 
46 Baudin-Baillieu, A. et al. Genome-wide translational changes induced by the prion [PSI+]. Cell Rep 8, 439-448, doi:10.1016/j.celrep.2014.06.036 (2014).

47 True, H. L., Berlin, I. \& Lindquist, S. L. Epigenetic regulation of translation reveals hidden genetic variation to produce complex traits. Nature 431, 184-187 (2004).

48 Tyedmers, J., Madariaga, M. L. \& Lindquist, S. Prion switching in response to environmental stress. PLoS Biol 6, e294, doi:10.1371/journal.pbio.0060294 (2008).

49 Halfmann, R. et al. Prions are a common mechanism for phenotypic inheritance in wild yeasts. Nature 482, 363-368, doi:10.1038/nature10875 (2012).

50 Yuan, A. H. \& Hochschild, A. A bacterial global regulator forms a prion. Science 355, 198201, doi:10.1126/science.aai7776 (2017).

The discovery of the first prokaryotic prion, the transcription terminator Rho of Clostridium

\section{botulinum.}

51 Chakrabortee, S. et al. Luminidependens (LD) is an Arabidopsis protein with prion behavior. Proc Natl Acad Sci U S A 113, 6065-6070, doi:10.1073/pnas.1604478113 (2016).

52 Jarosz, D. F. et al. Cross-kingdom chemical communication drives a heritable, mutually beneficial prion-based transformation of metabolism. Cell 158, 1083-1093, doi:10.1016/j.cell.2014.07.025 (2014).

53 Newby, G. A. \& Lindquist, S. Pioneer cells established by the [SWI+] prion can promote dispersal and out-crossing in yeast. PLoS Biol 15, e2003476, doi:10.1371/journal.pbio.2003476 (2017).

A lucid description of the mechanism by which the yeast prion [SWI ] confers a selective advantage. It enhances cells' ability to disperse in water and increases the likelihood of mating with dissimilar partners, thus facilitating migration, the colonization of new habitats, and genetic diversification.

54 Suzuki, G., Shimazu, N. \& Tanaka, M. A yeast prion, Mod5, promotes acquired drug resistance and cell survival under environmental stress. Science 336, 355-359, doi:10.1126/science.1219491 (2012).

55 Chakrabortee, S. et al. Intrinsically Disordered Proteins Drive Emergence and Inheritance of Biological Traits. Cell 167, 369-381 e312, doi:10.1016/j.cell.2016.09.017 (2016).

56 Franzmann, T. M. et al. Phase separation of a yeast prion protein promotes cellular fitness. Science 359, doi:10.1126/science.aao5654 (2018).

57 Riback, J. A. et al. Stress-Triggered Phase Separation Is an Adaptive, Evolutionarily Tuned Response. Cell 168, 1028-1040 e1019, doi:10.1016/j.cell.2017.02.027 (2017).

58 Avery, S. V. Microbial cell individuality and the underlying sources of heterogeneity. Nat Rev Microbiol 4, 577-587, doi:10.1038/nrmicro1460 (2006).

59 Flavahan, W. A., Gaskell, E. \& Bernstein, B. E. Epigenetic plasticity and the hallmarks of cancer. Science 357, doi:10.1126/science.aal2380 (2017).

60 Torres, C. M. et al. The linker histone H1.0 generates epigenetic and functional intratumor heterogeneity. Science 353, doi:10.1126/science.aaf1644 (2016).

This study provides a mechanistic account of how an epigenetic modification to a regulatory element

creates tumour-maintaining cells.

61 Aharoni, A. et al. The 'evolvability' of promiscuous protein functions. Nat Genet 37, 73-76, doi:10.1038/ng1482 (2005).

62 Tokuriki, N. \& Tawfik, D. S. Protein dynamism and evolvability. Science 324, 203-207, doi:10.1126/science.1169375 (2009). 
63 Copley, S. D. Enzymes with extra talents: moonlighting functions and catalytic promiscuity. Curr Opin Chem Biol 7, 265-272 (2003).

64 Notebaart, R. A. et al. Network-level architecture and the evolutionary potential of underground metabolism. Proc Natl Acad Sci U S A 111, 11762-11767, doi:10.1073/pnas.1406102111 (2014).

Pougach, K. et al. Duplication of a promiscuous transcription factor drives the emergence of a new regulatory network. Nat Commun 5, 4868, doi:10.1038/ncomms5868 (2014). Sayou, C. et al. A promiscuous intermediate underlies the evolution of LEAFY DNA binding specificity. Science 343, 645-648, doi:10.1126/science.1248229 (2014).

67 Petrie, K. L. et al. Destabilizing mutations encode nongenetic variation that drives evolutionary innovation. Science 359, 1542-1545, doi:10.1126/science.aar1954 (2018).

68 Rebeiz, M., Jikomes, N., Kassner, V. A. \& Carroll, S. B. Evolutionary origin of a novel gene expression pattern through co-option of the latent activities of existing regulatory sequences. Proc Natl Acad Sci U S A 108, 10036-10043, doi:10.1073/pnas.1105937108 (2011).

69 Wagner, A. Robustness and evolvability in living systems., (Princeton University Press, 2005).

70 Fares, M. A. The origins of mutational robustness. Trends Genet 31, 373-381, doi:10.1016/j.tig.2015.04.008 (2015).

71 Bloom, J. D., Labthavikul, S. T., Otey, C. R. \& Arnold, F. H. Protein stability promotes evolvability. Proc Natl Acad Sci U S A 103, 5869-5874, doi:10.1073/pnas.0510098103 (2006).

72 Ingolia, N. T. Topology and robustness in the Drosophila segment polarity network. PLoS Biol 2, e123, doi:10.1371/journal.pbio.0020123 (2004).

73 Segre, D., Vitkup, D. \& Church, G. Analysis of optimality in natural and perturbed metabolic networks. Proceedings of the National Academy of Sciences of the U.S.A. 99, 15112-15117 (2002).

74 Keane, O. M., Toft, C., Carretero-Paulet, L., Jones, G. W. \& Fares, M. A. Preservation of genetic and regulatory robustness in ancient gene duplicates of Saccharomyces cerevisiae. Genome Res 24, 1830-1841, doi:10.1101/gr.176792.114 (2014).

75 Diss, G. et al. Gene duplication can impart fragility, not robustness, in the yeast protein interaction network. Science 355, 630-634, doi:10.1126/science.aai7685 (2017).

76 Baker, C. R., Hanson-Smith, V. \& Johnson, A. D. Following gene duplication, paralog interference constrains transcriptional circuit evolution. Science 342, 104-108, doi:10.1126/science. 1240810 (2013).

77 Aguilar-Rodriguez, J. et al. The Molecular Chaperone DnaK Is a Source of Mutational Robustness. Genome Biol Evol 8, 2979-2991, doi:10.1093/gbe/evw176 (2016).

78 Jarosz, D. F. \& Lindquist, S. Hsp90 and environmental stress transform the adaptive value of natural genetic variation. Science 330, 1820-1824, doi:10.1126/science.1195487 (2010). Queitsch, C., Sangster, T. A. \& Lindquist, S. Hsp90 as a capacitor of phenotypic variation. Nature 417, 618-624, doi:DOI 10.1038/nature749 (2002).

80 Rohner, N. et al. Cryptic Variation in Morphological Evolution: HSP90 as a Capacitor for Loss of Eyes in Cavefish. Science 342, 1372-1375, doi:10.1126/science.1240276 (2013).

81 Rutherford, S. L. \& Lindquist, S. Hsp90 as a capacitor for morphological evolution. Nature 396, 336-342, doi:10.1038/24550 (1998).

82 Tokuriki, N. \& Tawfik, D. S. Chaperonin overexpression promotes genetic variation and enzyme evolution. Nature 459, 668-673 (2009).

83 Geiler-Samerotte, K. A., Zhu, Y. O., Goulet, B. E., Hall, D. W. \& Siegal, M. L. Selection transforms the landscape of genetic variation interacting with Hsp90. Plos Biology 14, e2000465 (2016). 
84 Stiffler, M. A., Hekstra, D. R. \& Ranganathan, R. Evolvability as a function of purifying selection in TEM-1 beta-lactamase. Cell 160, 882-892, doi:10.1016/j.cell.2015.01.035 (2015).

This study showed that the synergism between mutational robustness and evolvability in TEM-1 $\beta$ -

lactamase depends upon the strength of purifying selection for ampicillin resistance.

85 Masel, J. \& Bergman, A. The evolution of the evolvability properties of the yeast prion [PSI+]. Evolution 57, 1498-1512 (2003).

86 Hayden, E. J., Ferrada, E. \& Wagner, A. Cryptic genetic variation promotes rapid evolutionary adaptation in an RNA enzyme. Nature 474, 92-95, doi:10.1038/nature10083 (2011).

87 Payne, J. L. \& Wagner, A. The robustness and evolvability of transcription factor binding sites. Science 343, 875-877, doi:10.1126/science.1249046 (2014).

88 de Visser, J. A. et al. Perspective: Evolution and detection of genetic robustness. Evolution 57, 1959-1972 (2003).

89 Najafabadi, H. S. et al. Non-base-contacting residues enable kaleidoscopic evolution of metazoan $\mathrm{C} 2 \mathrm{H} 2$ zinc finger DNA binding. Genome Biol 18, 167, doi:10.1186/s13059-0171287-y (2017).

A mechanistic explanation of the expansion and diversification of metazoan $\mathrm{C} 2 \mathrm{H} 2$ zinc-finger transcription factors.

90 McKeown, A. N. et al. Evolution of DNA specificity in a transcription factor family produced a new gene regulatory module. Cell 159, 58-68, doi:10.1016/j.cell.2014.09.003 (2014).

91 Starr, T. N., Picton, L. K. \& Thornton, J. W. Alternative evolutionary histories in the sequence space of an ancient protein. Nature 549, 409-413, doi:10.1038/nature23902 (2017).

92 Payne, J. L., Khalid, F. \& Wagner, A. RNA-mediated gene regulation is less evolvable than transcriptional regulation. Proc Natl Acad Sci U S A 115, E3481-E3490, doi:10.1073/pnas.1719138115 (2018).

93 Berger, M. F. et al. Compact, universal DNA microarrays to comprehensively determine transcription-factor binding site specificities. Nat Biotechnol 24, 1429-1435, doi:10.1038/nbt1246 (2006).

94 Aguilar-Rodriguez, J., Payne, J. L. \& Wagner, A. A thousand empirical adaptive landscapes and their navigability. Nat Ecol Evol 1, 45, doi:10.1038/s41559-016-0045 (2017).

95 Weirauch, M. T. \& Hughes, T. R. Conserved expression without conserved regulatory sequence: the more things change, the more they stay the same. Trends Genet 26, 66-74, doi:10.1016/j.tig.2009.12.002 (2010).

96 Tsong, A. E., Tuch, B. B., Li, H. \& Johnson, A. D. Evolution of alternative transcriptional circuits with identical logic. Nature 443, 415-420, doi:10.1038/nature05099 (2006).

97 Ciliberti, S., Martin, O. C. \& Wagner, A. Innovation and robustness in complex regulatory gene networks. Proc Natl Acad Sci U S A 104, 13591-13596, doi:10.1073/pnas.0705396104 (2007).

98 Martin, O. C. \& Wagner, A. Effects of recombination on complex regulatory circuits. Genetics 183, 673-684, 671SI-678SI, doi:10.1534/genetics.109.104174 (2009).

99 Nocedal, I., Mancera, E. \& Johnson, A. D. Gene regulatory network plasticity predates a switch in function of a conserved transcription regulator. Elife 6, doi:10.7554/eLife. 23250 (2017). 
100 Doud, M. B. \& Bloom, J. D. Accurate Measurement of the Effects of All Amino-Acid Mutations on Influenza Hemagglutinin. Viruses-Basel 8, doi:ARTN 155

10.3390/v8060155 (2016).

101 Haddox, H. K., Dingens, A. S. \& Bloom, J. D. Experimental Estimation of the Effects of All Amino-Acid Mutations to HIV's Envelope Protein on Viral Replication in Cell Culture. Plos Pathog 12, doi:ARTN e1006114

10.1371/journal.ppat.1006114 (2016).

102 Jacquier, H. et al. Capturing the mutational landscape of the beta-lactamase TEM-1. P Natl Acad Sci USA 110, 13067-13072, doi:10.1073/pnas.1215206110 (2013).

103 Findlay, G. M., Boyle, E. A., Hause, R. J., Klein, J. C. \& Shendure, J. Saturation editing of genomic regions by multiplex homology-directed repair. Nature 513, 120-+, doi:10.1038/nature13695 (2014).

104 Podgornaia, A. I. \& Laub, M. T. Protein evolution. Pervasive degeneracy and epistasis in a protein-protein interface. Science 347, 673-677, doi:10.1126/science.1257360 (2015).

105 Aakre, C. D. et al. Evolving New Protein-Protein Interaction Specificity through Promiscuous Intermediates. Cell 163, 594-606, doi:10.1016/j.cell.2015.09.055 (2015).

106 Crombach, A., Wotton, K. R., Jimenez-Guri, E. \& Jaeger, J. Gap Gene Regulatory Dynamics Evolve along a Genotype Network. Molecular Biology and Evolution 33, 1293-1307, doi:10.1093/molbev/msw013 (2016).

107 Smith, J. M. Natural selection and the concept of a protein space. Nature 225, 563-564 (1970).

108 Wright, S. The roles of mutation, inbreeding, crossbreeding and selection in evolution. Proc Sixth Internat Congr Genetics Ithaca New York 1, 356-366 (1932).

109 de Visser, J. A. \& Krug, J. Empirical fitness landscapes and the predictability of evolution. Nat Rev Genet 15, 480-490, doi:10.1038/nrg3744 (2014).

110 Li, C., Qian, W., Maclean, C. J. \& Zhang, J. The fitness landscape of a tRNA gene. Science 352, 837-840, doi:10.1126/science.aae0568 (2016).

111 Puchta, O. et al. Network of epistatic interactions within a yeast snoRNA. Science 352, 840844, doi:10.1126/science.aaf0965 (2016).

References 110 and 111 are, at present, the highest-resolution characterizations of local adaptive

landscape topography, where the landscape surface represents organismal fitness.

112 Qiu, C. et al. High-Resolution Phenotypic Landscape of the RNA Polymerase II Trigger Loop. PLoS Genet 12, e1006321, doi:10.1371/journal.pgen.1006321 (2016).

113 Wrenbeck, E. E., Azouz, L. R. \& Whitehead, T. A. Single-mutation fitness landscapes for an enzyme on multiple substrates reveal specificity is globally encoded. Nat Commun 8, 15695, doi:10.1038/ncomms15695 (2017).

114 Melamed, D., Young, D. L., Gamble, C. E., Miller, C. R. \& Fields, S. Deep mutational scanning of an RRM domain of the Saccharomyces cerevisiae poly (A)-binding protein. $R N A$ 19, 1537-1551, doi:10.1261/rna.040709.113 (2013).

115 Olson, C. A., Wu, N. C. \& Sun, R. A comprehensive biophysical description of pairwise epistasis throughout an entire protein domain. Curr Biol 24, 2643-2651, doi:10.1016/j.cub.2014.09.072 (2014).

116 Julien, P., Minana, B., Baeza-Centurion, P., Valcarcel, J. \& Lehner, B. The complete local genotype-phenotype landscape for the alternative splicing of a human exon. Nat Commun 7, 11558, doi:10.1038/ncomms11558 (2016).

117 Poelwijk, F. J., Kiviet, D. J., Weinreich, D. M. \& Tans, S. J. Empirical fitness landscapes reveal accessible evolutionary paths. Nature 445, 383-386, doi:10.1038/nature05451 (2007). 
118 Poelwijk, F. J., Tanase-Nicola, S., Kiviet, D. J. \& Tans, S. J. Reciprocal sign epistasis is a necessary condition for multi-peaked fitness landscapes. J Theor Biol 272, 141-144, doi:10.1016/j.jtbi.2010.12.015 (2011).

119 Chou, H. H., Chiu, H. C., Delaney, N. F., Segre, D. \& Marx, C. J. Diminishing returns epistasis among beneficial mutations decelerates adaptation. Science 332, 1190-1192, doi:10.1126/science.1203799 (2011).

120 Khan, A. I., Dinh, D. M., Schneider, D., Lenski, R. E. \& Cooper, T. F. Negative epistasis between beneficial mutations in an evolving bacterial population. Science 332, 1193-1196, doi:10.1126/science.1203801 (2011).

121 Elena, S. F. \& Lenski, R. E. Test of synergistic interactions among deleterious mutations in bacteria. Nature 390, 395-398, doi:Doi 10.1038/37108 (1997).

122 Weinreich, D. M., Watson, R. A. \& Chao, L. Perspective: Sign epistasis and genetic constraint on evolutionary trajectories. Evolution 59, 1165-1174 (2005).

123 Weinreich, D. M. \& Chao, L. Rapid evolutionary escape by large populations from local fitness peaks is likely in nature. Evolution 59, 1175-1182 (2005).

124 Iwasa, Y., Michor, F. \& Nowak, M. A. Stochastic tunnels in evolutionary dynamics. Genetics 166, 1571-1579 (2004).

125 Anderson, D. W., McKeown, A. N. \& Thornton, J. W. Intermolecular epistasis shaped the function and evolution of an ancient transcription factor and its DNA binding sites. Elife 4, e07864, doi:10.7554/elife.07864 (2015).

126 Lagator, M., Sarikas, S., Acar, H., Bollback, J. P. \& Guet, C. C. Regulatory network structure determines patterns of intermolecular epistasis. Elife 6, doi:10.7554/eLife.28921 (2017).

References 125 and 126 showed that inter-molecular sign epistasis can facilitate, rather than impede, evolvability.

127 Palmer, A. C. et al. Delayed commitment to evolutionary fate in antibiotic resistance fitness landscapes. Nat Commun 6, 7385, doi:10.1038/ncomms8385 (2015).

128 Buckling, A., Maclean, R. C., Brockhurst, M. A. \& Colegrave, N. The Beagle in a bottle. Nature 457, 824-829 (2009).

129 Jerison, E. R. et al. Genetic variation in adaptability and pleiotropy in budding yeast. Elife 6, doi:10.7554/eLife.27167 (2017).

130 Kryazhimskiy, S., Rice, D. P., Jerison, E. R. \& Desai, M. M. Microbial evolution. Global epistasis makes adaptation predictable despite sequence-level stochasticity. Science 344, 1519-1522, doi:10.1126/science.1250939 (2014).

131 Wunsche, A. et al. Diminishing-returns epistasis decreases adaptability along an evolutionary trajectory. Nat Ecol Evol 1, 61, doi:10.1038/s41559-016-0061 (2017).

132 Wiser, M. J., Ribeck, N. \& Lenski, R. E. Long-term dynamics of adaptation in asexual populations. Science 342, 1364-1367, doi:10.1126/science.1243357 (2013).

133 Good, B. H. \& Desai, M. M. The impact of macroscopic epistasis on long-term evolutionary dynamics. Genetics 199, 177-190, doi:10.1534/genetics.114.172460 (2015).

134 Kryazhimskiy, S., Tkacik, G. \& Plotkin, J. B. The dynamics of adaptation on correlated fitness landscapes. Proc Natl Acad Sci U S A 106, 18638-18643, doi:10.1073/pnas.0905497106 (2009).

135 Gifford, D. R., Toll-Riera, M. \& MacLean, R. C. Epistatic interactions between ancestral genotype and beneficial mutations shape evolvability in Pseudomonas aeruginosa. Evolution 70, 1659-1666, doi:10.1111/evo.12958 (2016).

136 Good, B. H., McDonald, M. J., Barrick, J. E., Lenski, R. E. \& Desai, M. M. The dynamics of molecular evolution over 60,000 generations. Nature 551, 45-50, doi:10.1038/nature24287 (2017). 
137 Venkataram, S. et al. Development of a Comprehensive Genotype-to-Fitness Map of Adaptation-Driving Mutations in Yeast. Cell 166, 1585-1596 e1522, doi:10.1016/j.cell.2016.08.002 (2016).

138 Bank, C., Matuszewski, S., Hietpas, R. T. \& Jensen, J. D. On the (un)predictability of a large intragenic fitness landscape. Proc Natl Acad Sci U S A 113, 14085-14090, doi:10.1073/pnas.1612676113 (2016).

139 Fowler, D. M. \& Fields, S. Deep mutational scanning: a new style of protein science. Nat Methods 11, 801-807, doi:10.1038/nmeth.3027 (2014).

140 Sarkisyan, K. S. et al. Local fitness landscape of the green fluorescent protein. Nature 533, 397-401, doi:10.1038/nature17995 (2016).

141 Bank, C., Hietpas, R. T., Jensen, J. D. \& Bolon, D. N. A systematic survey of an intragenic epistatic landscape. Mol Biol Evol 32, 229-238, doi:10.1093/molbev/msu301 (2015).

142 Bershtein, S., Segal, M., Bekerman, R., Tokuriki, N. \& Tawfik, D. S. Robustness-epistasis link shapes the fitness landscape of a randomly drifting protein. Nature 444, 929-932, doi:10.1038/nature05385 (2006).

143 Trindade, S. et al. Positive epistasis drives the acquisition of multidrug resistance. PLoS Genet 5, e1000578, doi:10.1371/journal.pgen.1000578 (2009).

144 Steinberg, B. \& Ostermeier, M. Environmental changes bridge evolutionary valleys. Sci Adv 2, e1500921, doi:10.1126/sciadv.1500921 (2016).

145 du Plessis, L., Leventhal, G. E. \& Bonhoeffer, S. How Good Are Statistical Models at Approximating Complex Fitness Landscapes? Mol Biol Evol 33, 2454-2468, doi:10.1093/molbev/msw097 (2016).

146 Otwinowski, J. \& Plotkin, J. B. Inferring fitness landscapes by regression produces biased estimates of epistasis. Proc Natl Acad Sci U S A 111, E2301-2309, doi:10.1073/pnas.1400849111 (2014).

147 Jimenez, J. I., Xulvi-Brunet, R., Campbell, G. W., Turk-MacLeod, R. \& Chen, I. A. Comprehensive experimental fitness landscape and evolutionary network for small RNA. Proc Natl Acad Sci U S A 110, 14984-14989, doi:10.1073/pnas.1307604110 (2013).

148 Rowe, W. et al. Analysis of a complete DNA-protein affinity landscape. J R Soc Interface 7, 397-408, doi:10.1098/rsif.2009.0193 (2010).

149 Weirauch, M. T. et al. Determination and inference of eukaryotic transcription factor sequence specificity. Cell 158, 1431-1443, doi:10.1016/j.cell.2014.08.009 (2014).

150 Sharon, E. et al. Inferring gene regulatory logic from high-throughput measurements of thousands of systematically designed promoters. Nature Biotechnology 30, 521-+, doi:10.1038/nbt.2205 (2012).

151 Shultzaberger, R. K., Malashock, D. S., Kirsch, J. F. \& Eisen, M. B. The Fitness Landscapes of cis-Acting Binding Sites in Different Promoter and Environmental Contexts. Plos Genetics 6, doi:ARTN e1001042

10.1371/journal.pgen.1001042 (2010).

152 Gavrilets, S. Evolution and speciation on holey adaptive landscapes. Trends in Ecology \& Evolution 12, 307-312, doi:Doi 10.1016/S0169-5347(97)01098-7 (1997).

153 Wu, N. C., Dai, L., Olson, C. A., Lloyd-Smith, J. O. \& Sun, R. Adaptation in protein fitness landscapes is facilitated by indirect paths. Elife 5, doi:10.7554/eLife.16965 (2016).

154 Hartman, E. C. et al. Quantitative characterization of all single amino acid variants of a viral capsid-based drug delivery vehicle. Nature Communications 9, doi:ARTN 1385

10.1038/s41467-018-03783-y (2018).

155 Rogers, Z. N. et al. Mapping the in vivo fitness landscape of lung adenocarcinoma tumor suppression in mice. Nature Genetics 50, 483-+, doi:10.1038/s41588-018-0083-2 (2018).

156 Sniegowski, P. D., Gerrish, P. J. \& Lenski, R. E. Evolution of high mutation rates in experimental populations of E. coli. Nature 387, 703-705, doi:10.1038/42701 (1997). 
157 Wielgoss, S. et al. Mutation rate dynamics in a bacterial population reflect tension between adaptation and genetic load. Proc Natl Acad Sci U S A 110, 222-227,

doi:10.1073/pnas.1219574110 (2013).

158 Healey, K. R. et al. Prevalent mutator genotype identified in fungal pathogen Candida glabrata promotes multi-drug resistance. Nat Commun 7, 11128, doi:10.1038/ncomms11128 (2016).

159 Giraud, A. et al. Costs and benefits of high mutation rates: adaptive evolution of bacteria in the mouse gut. Science 291, 2606-2608 (2001).

160 McDonald, M. J., Rice, D. P. \& Desai, M. M. Sex speeds adaptation by altering the dynamics of molecular evolution. Nature 531, 233-236, doi:10.1038/nature17143 (2016).

161 Rego, E. H., Audette, R. E. \& Rubin, E. J. Deletion of a mycobacterial divisome factor collapses single-cell phenotypic heterogeneity. Nature 546, 153-157, doi:10.1038/nature22361 (2017).

This study showed that heterogeneity of cell growth in mycobacteria is partially controlled by LamA, a

divisome factor responsible for asymmetric growth of daughter cells after cell division.

162 Jones, D. L., Brewster, R. C. \& Phillips, R. Promoter architecture dictates cell-to-cell variability in gene expression. Science 346, 1533-1536, doi:10.1126/science.1255301 (2014).

163 Dabrowski, M., Bukowy-Bieryllo, Z. \& Zietkiewicz, E. Translational readthrough potential of natural termination codons in eucaryotes - The impact of RNA sequence. Rna Biol 12, 950958, doi:10.1080/15476286.2015.1068497 (2015).

164 Paul, K. R., Hendrich, C. G., Waechter, A., Harman, M. R. \& Ross, E. D. Generating new prions by targeted mutation or segment duplication. Proc Natl Acad Sci U S A 112, 85848589, doi:10.1073/pnas.1501072112 (2015).

165 Zhang, Z., Qian, W. \& Zhang, J. Positive selection for elevated gene expression noise in yeast. Mol Syst Biol 5, 299, doi:10.1038/msb.2009.58 (2009).

166 Wolf, L., Silander, O. K. \& van Nimwegen, E. Expression noise facilitates the evolution of gene regulation. Elife 4, doi:10.7554/eLife.05856 (2015).

167 Osterwalder, M. et al. Enhancer redundancy provides phenotypic robustness in mammalian development. Nature 554, 239-243, doi:10.1038/nature25461 (2018).

168 Borenstein, E. \& Ruppin, E. Direct evolution of genetic robustness in microRNA. Proc Natl Acad Sci U S A 103, 6593-6598, doi:10.1073/pnas.0510600103 (2006).

169 Toll-Riera, M., Bostick, D., Alba, M. M. \& Plotkin, J. B. Structure and age jointly influence rates of protein evolution. PLoS Comput Biol 8, e1002542, doi:10.1371/journal.pcbi.1002542 (2012).

170 Bloom, J. D. et al. Evolution favors protein mutational robustness in sufficiently large populations. BMC Biol 5, 29, doi:10.1186/1741-7007-5-29 (2007).

Experimental demonstration that mutational robustness increases in the evolution of large populations.

171 Montville, R., Froissart, R., Remold, S. K., Tenaillon, O. \& Turner, P. E. Evolution of mutational robustness in an RNA virus. PLoS Biol 3, e381, doi:10.1371/journal.pbio.0030381 (2005).

172 Sanjuan, R., Cuevas, J. M., Furio, V., Holmes, E. C. \& Moya, A. Selection for robustness in mutagenized RNA viruses. PLoS Genet 3, e93, doi:10.1371/journal.pgen.0030093 (2007).

173 Szollosi, G. J. \& Derenyi, I. Congruent evolution of genetic and environmental robustness in micro-RNA. Mol Biol Evol 26, 867-874, doi:10.1093/molbev/msp008 (2009).

174 Salverda, M. L. et al. Initial mutations direct alternative pathways of protein evolution. PLoS Genet 7, e1001321, doi:10.1371/journal.pgen.1001321 (2011). 
175 Chan, Y. H., Venev, S. V., Zeldovich, K. B. \& Matthews, C. R. Correlation of fitness landscapes from three orthologous TIM barrels originates from sequence and structure constraints. Nat Commun 8, 14614, doi:10.1038/ncomms14614 (2017).

176 Haddox, H. K., Dingens, A. S., Hilton, S. K., Overbaugh, J. \& Bloom, J. D. Mapping mutational effects along the evolutionary landscape of HIV envelope. Elife 7, doi: $10.7554 /$ eLife.34420 (2018).

177 Woods, R. J. et al. Second-order selection for evolvability in a large Escherichia coli population. Science 331, 1433-1436, doi:10.1126/science.1198914 (2011).

178 Leon, D., D'Alton, S., Quandt, E. M. \& Barrick, J. E. Innovation in an E. coli evolution experiment is contingent on maintaining adaptive potential until competition subsides. PLoS Genet 14, e1007348, doi:10.1371/journal.pgen.1007348 (2018).

179 Gommans, W. M., Mullen, S. P. \& Maas, S. RNA editing: a driving force for adaptive evolution? Bioessays 31, 1137-1145, doi:10.1002/bies.200900045 (2009).

180 Raman, A. S., White, K. I. \& Ranganathan, R. Origins of Allostery and Evolvability in Proteins: A Case Study. Cell 166, 468-480, doi:10.1016/j.cell.2016.05.047 (2016).

181 Domingo, J., Diss, G. \& Lehner, B. Pairwise and higher-order genetic interactions during the evolution of a tRNA. Nature 558, 117-121, doi:10.1038/s41586-018-0170-7 (2018).

182 Sailer, Z. R. \& Harms, M. J. High-order epistasis shapes evolutionary trajectories. PLoS Comput Biol 13, e1005541, doi:10.1371/journal.pcbi.1005541 (2017).

183 de Vos, M. G., Dawid, A., Sunderlikova, V. \& Tans, S. J. Breaking evolutionary constraint with a tradeoff ratchet. Proc Natl Acad Sci U S A 112, 14906-14911, doi:10.1073/pnas.1510282112 (2015).

References 144 and 183 demonstrate that environmental change can facilitate adaptation by helping evolving populations escape local optima of an adaptive landscape.

184 Ogbunugafor, C. B. \& Eppstein, M. J. Competition along trajectories governs adaptation rates towards antimicrobial resistance. Nat Ecol Evol 1, 7, doi:10.1038/s41559-016-0007 (2016).

185 Moratorio, G. et al. Attenuation of RNA viruses by redirecting their evolution in sequence space. Nat Microbiol 2, 17088, doi:10.1038/nmicrobiol.2017.88 (2017).

186 Prakadan, S. M., Shalek, A. K. \& Weitz, D. A. Scaling by shrinking: empowering single-cell 'omics' with microfluidic devices. Nat Rev Genet 18, 345-361, doi:10.1038/nrg.2017.15 (2017).

187 Crosetto, N., Bienko, M. \& van Oudenaarden, A. Spatially resolved transcriptomics and beyond. Nat Rev Genet 16, 57-66, doi:10.1038/nrg3832 (2015).

188 Liu, S. \& Trapnell, C. Single-cell transcriptome sequencing: recent advances and remaining challenges. F1000Res 5, doi:10.12688/f1000research.7223.1 (2016).

189 Ingolia, N. T. Ribosome Footprint Profiling of Translation throughout the Genome. Cell 165, 22-33, doi:10.1016/j.cell.2016.02.066 (2016).

190 Hietpas, R. T., Jensen, J. D. \& Bolon, D. N. Experimental illumination of a fitness landscape. Proc Natl Acad Sci U S A 108, 7896-7901, doi:10.1073/pnas.1016024108 (2011).

191 Thornton, J. W. Resurrecting ancient genes: experimental analysis of extinct molecules. Nat Rev Genet 5, 366-375, doi:10.1038/nrg1324 (2004).

192 Eyre-Walker, A. \& Keightley, P. D. The distribution of fitness effects of new mutations. Nature Reviews Genetics 8, 610-618 (2007).

193 Eshel, I. Clone-selection and optimal rates of mutation. Journal of Applied Probability 10, 728-738 (1973).

194 Starrfelt, J. \& Kokko, H. Bet-hedging-a triple trade-off between means, variances and correlations. Biological Reviews 87, 742-755 (2012).

195 Frank, S. A. Foundations of social evolution. (Princeton University Press, 1998). 
196 Gardner, A., West, S. A. \& Wild, G. The genetical theory of kin selection. Journal of Evolutionary Biology 24, 1020-1043, doi:10.1111/j.1420-9101.2011.02236.x (2011).

197 Wilson, D. S. \& Wilson, E. O. Rethinking the theoretical foundation of sociobiology. Quarterly Review of Biology 82, 327-348, doi:10.1086/522809 (2007).

198 Graves, C. J. \& Weinreich, D. M. Variability in Fitness Effects Can Preclude Selection of the Fittest. Annu Rev Ecol Evol S 48, 399-417, doi:10.1146/annurev-ecolsys-110316-022722 (2017).

199 Griswold, C. K. \& Masel, J. Complex adaptations can drive the evolution of the capacitor PSI+, even with realistic rates of yeast sex. Plos Genetics 5, doi:e1000517

10.1371/journal.pgen.1000517 (2009).

200 Rutherford, S. L. Between genotype and phenotype: protein chaperones and evolvability. Nat Rev Genet 4, 263-274, doi:10.1038/nrg1041 (2003).

\section{Competing interests}

The authors declare no competing interests.

\section{Subject categories}

\section{Biological sciences / Genetics / Evolutionary biology}

[URI /631/208/182]

\section{Biological sciences / Evolution / Molecular evolution}

[URI /631/181/735]

\section{Biological sciences / Evolution / Evolutionary genetics}

[URI /631/181/2474]

\section{Biological sciences / Evolution / Experimental evolution}

[URI /631/181/2475]

Biological sciences / Systems biology / Robustness

[URI /631/553/2715] 
[URI /631/553/2716]

Table of contents blurb

In this article, Payne and Wagner discuss how recent experimental studies are complementing theoretical work to enhance our understanding of the evolvability of diverse biological systems. They highlight phenotypic heterogeneity, robustness and adaptive landscape topography as causes of evolvability, and they additionally discuss evidence for whether evolvability itself can evolve. 